\title{
A Mortar Mimetic Finite Difference Method on Non-Matching Grids
}

\author{
Markus Berndt* Konstantin Lipnikov* Mikhail Shashkov* \\ Mary F. Wheeler ${ }^{\dagger} \quad$ Ivan Yotov ${ }^{\ddagger}$
}

July 26, 2004

\begin{abstract}
We consider mimetic finite difference approximations to second order elliptic problems on non-matching multi-block grids. Mortar finite elements are employed on the non-matching interfaces to impose weak continuity of the velocity. Optimal convergence and, for certain cases, superconvergence is established for both the scalar variable and the velocity.
\end{abstract}

\section{Introduction}

In this work, we consider second order linear elliptic equations that in porous medium applications model single phase Darcy flow. We solve for pressure $p$ and velocity $\mathbf{u}$ satisfying

$$
\begin{array}{ll}
\mathbf{u}=-K \nabla p & \text { in } \Omega, \\
\nabla \cdot \mathbf{u}=b & \text { in } \Omega, \\
p=g & \text { on } \partial \Omega,
\end{array}
$$

where $\Omega \subset \mathbb{R}^{\operatorname{dim}}$, $\operatorname{dim}=2$ or 3 , is a multi-block domain, and $K$ is a symmetric, uniformly positive definite tensor with $L^{\infty}(\Omega)$ components representing the permeability divided by the viscosity. The Dirichlet boundary conditions are considered merely for simplicity.

In this paper, we analyze the convergence of a mortar mimetic finite difference method (mortar MFD) on quadrilateral and simplicial meshes. The MFD method employs discrete operators that preserve certain critical properties of the original continuum differential operators, such as conservation laws, solution symmetries, and fundamental identities of vector calculus. The mimetic technique has been successfully employed in a number of applications, including diffusion [19, 12, 15], continuum mechanics [14], and gas dynamics [8]. It

*Los Alamos National Laboratory, Mail Stop B284, Los Alamos, NM 87545, U.S.A., \{berndt, lipnikov, shashkov\}@lanl.gov. Supported by the U.S. Department of Energy, under contract W-7405-ENG-36. LA-UR-04-4740.

${ }^{\dagger}$ Institute for Computational Engineering and Sciences (ICES), Department of Aerospace Engineering and Engineering Mechanics, and Department of Petroleum and Geosystems Engineering, The University of Texas at Austin, Austin, TX 78712, U.S.A., mfw@ices.utexas.edu. Partially supported by NSF grant EIA-0121523 and by NPACI grant UCSD 10181410.

${ }_{\ddagger}^{\ddagger}$ Department of Mathematics, 301 Thackeray Hall, University of Pittsburgh, Pittsburgh, PA 15260, U.S.A., yotov@math.pitt.edu. Partially supported by NSF grants DMS 0107389, DMS 0112239 and DMS 0411694. 
has been extended to locally refined meshes with hanging nodes [13], and unstructured threedimensional meshes comprised of hexahedrons, tetrahedrons, and any cell type that has three faces intersecting at each vertex. In this paper, we employ mortar finite element techniques to extend the MFD method to the case of non-matching multi-block mortar grids.

Mixed finite element (MFE) discretizations on quadrilateral meshes [20, 21, 2, 10] are based on the Piola transformation [20, 7], which preserves continuity of the normal component of the velocity across mesh edges, but results in the necessity to integrate rational functions over quadrilaterals. This is further complicated in the case of a full or non-constant diffusion tensor. The results in [5] provide an efficient numerical quadrature with a minimal number of points, allowing for the extension of MFE methods to general polygons and polyhedra.

The mortar MFE method has been studied, for example, in [23, 1] (see also [4, 3, 22] for seminal work on mortar couplings for Galerkin finite element discretizations). In these methods, the domain is divided into nonoverlapping subdomain blocks, and each of these subdomain blocks is discretized on a locally constructed mesh. As a result, the subdomain grids do not match at interblock boundaries. To solve this problem, Lagrange multiplier pressures are introduced at the interblock boundaries. This Lagrange multiplier space is called the mortar finite element space. It was shown in [1] that the mortar MFE method is optimally convergent, if the boundary space has one order higher approximability than the normal trace of the velocity space.

A connection between the MFD method and the MFE method with Raviart-Thomas finite elements was established in [5]. This was achieved by showing that the scalar product in the velocity space proposed in [12] for MFD methods can be viewed as a quadrature rule in the context of MFE methods. In [6], superconvergence for the normal velocities in MFD methods on $h^{2}$-uniform quadrilateral meshes is established. In this paper, we exploit the relation between the methods to show that the mortar MFD method exhibits optimal convergence. We also establish superconvergence for the normal velocity on $h^{2}$-uniform quadrilateral meshes and for the pressure on simplicial and general quadrilateral meshes.

The outline of the paper is as follows. In Section 2, we describe the mortar MFD method by extending the MFD method to the case of non-overlapping subdomain blocks. In Section 3, the mortar MFE method is described, and in Section 4 it is related to the mortar MFD method. In Sections 5 and 6, we give error estimates for the velocity and the pressure, respectively. We conclude the paper with numerical experiments in Section 7.

\section{Mortar mimetic finite difference method}

We assume that $\Omega$ can be decomposed into non-overlapping subdomain blocks $\Omega_{i}$,

$$
\bar{\Omega}=\bigcup_{i=1}^{n} \bar{\Omega}_{i}
$$

Denote by $\Gamma_{i, j}=\partial \Omega_{i} \cap \partial \Omega_{j}$ the interior block interfaces. Let

$$
\Gamma=\bigcup_{i, j=1}^{n} \Gamma_{i, j}, \quad \text { and } \quad \Gamma_{i}=\partial \Omega_{i} \cap \Gamma=\partial \Omega_{i} \backslash \partial \Omega .
$$

Let $\mathcal{T}_{h, i}$ be a conforming, shape-regular, quasi-uniform partition of $\Omega_{i}, 1 \leq i \leq n$ [9], allowing for $\mathcal{T}_{h, i}$ and $\mathcal{T}_{h, j}$ to be non-matching on $\Gamma_{i, j}$. We will consider simplicial elements in two and three dimensions as well as convex quadrilateral elements in two dimensions. 
Let $\mathcal{E}_{h, i, j}$ be the trace of mesh $\mathcal{T}_{h, i}$ on the interface $\Gamma_{i, j}$ and let $\mathcal{E}_{h, i}$ be the trace of $\mathcal{T}_{h, i}$ on $\partial \Omega_{i}$. Let $\tilde{\mathcal{E}}_{h, i, j}$ be another quasi-uniform partition of $\Gamma_{i, j}$. We postulate that $\tilde{\mathcal{E}}_{h, i, j} \equiv \tilde{\mathcal{E}}_{h, j, i}$. This partition will be used to impose interface matching conditions via mortar finite elements. Finally, let

$$
\mathcal{T}_{h}=\bigcup_{i=1}^{n} \mathcal{T}_{h, i}
$$

\subsection{Subdomain discretization}

We now consider one subdomain $\Omega_{i}$ and introduce operators $\mathcal{G}$ and $\mathcal{D}$ by

$$
\mathcal{G} p=-K \nabla p, \quad \mathcal{D} \mathbf{u}=\left\{\begin{array}{cll}
\nabla \cdot \mathbf{u} & \text { on } & \Omega_{i} \\
-\mathbf{u} \cdot \mathbf{n}_{i} & \text { on } & \partial \Omega_{i}
\end{array}\right.
$$

where $\mathbf{n}_{i}$ is the outer unit normal to $\partial \Omega_{i}$. We shall refer to $\mathcal{G}$ and $\mathcal{D}$ as the flux and extended divergence operators, respectively. Let us introduce the scalar products

$$
(\mathbf{u}, \mathbf{v})_{X_{i}}=\int_{\Omega_{i}} K^{-1} \mathbf{u} \cdot \mathbf{v} \mathrm{d} \mathbf{x} \quad \text { and } \quad(p, q)_{Q_{i}}=\int_{\Omega_{i}} p q \mathrm{~d} \mathbf{x}+\oint_{\partial \Omega_{i}} p q \mathrm{~d} s,
$$

in the space $X_{i}=L^{2}\left(\Omega_{i}\right)$ of velocities and in the space $Q_{i}=H^{1}\left(\Omega_{i}\right)$ of pressures, respectively. Using the above notations, the Gauss-Green formula is written as

$$
(\mathbf{u}, \mathcal{G} p)_{X_{i}}=(p, \mathcal{D} \mathbf{u})_{Q_{i}} .
$$

This expression states that the flux and extended divergence operators are adjoint to each other, i.e. $\mathcal{G}=\mathcal{D}^{*}$. In this section, we derive discrete operators that are adjoint to each other with respect to certain scalar products in the discrete velocity and pressure spaces. For the sake of simplicity, we omit subscript ' $i$ ' whenever this does not result in ambiguity.

The first step in the mimetic finite difference (MFD) method is to specify discrete degrees of freedom for the primary variables, pressure and velocity. The discrete pressure unknowns are defined at the centers of the mesh elements of $\mathcal{T}_{h, i}$ and at the centers of the boundary faces of $\mathcal{E}_{h, i}$ (edges in 2D). The discrete velocities are defined at the midpoints of mesh faces of $\mathcal{T}_{h, i}$ (edges in 2D) as normal components. In other words, a face-based unknown is a scalar and represents the orthogonal projection of a velocity vector onto the unit vector normal to the mesh face. The direction of the normal vector is a priori fixed.

The second step in the MFD method is to equip the spaces of discrete pressures and velocities with scalar products. We denote the vector space of discrete pressures by $Q_{i}^{d}$. The scalar product on this vector space $Q_{i}^{d}$ is given by

$$
[\vec{p}, \vec{q}]_{Q_{i}^{d}}=\sum_{E \in \mathcal{T}_{h, i}}|E| p_{E} q_{E}+\sum_{f \in \mathcal{E}_{h, i}}|f| p_{f} q_{f}
$$

where $|E|$ denotes the volume (area in 2D) of element $E,|f|$ denotes the area (lengh in 2D) of face $f$, and $p_{E}, p_{f}$ denote pressure components associated with $E$ and $f$, respectively. Denote by $Q_{i}^{d, 0}$ the vector space of only cell-based unknowns. The scalar product on $Q_{i}^{d, 0}$ is given by the first sum in (2.1).

We denote the vector space of face-based velocities by $X_{i}^{d}$. The scalar product on $X_{i}^{d}$ is given by

$$
[\vec{u}, \vec{v}]_{X_{i}^{d}}=\sum_{E \in \mathcal{T}_{i, h}}[\vec{u}, \vec{v}]_{X_{i}^{d}, E}
$$


where $[\vec{u}, \vec{v}]_{X_{i}^{d}, E}$ is a scalar product over element $E$ involving only normal velocity components on element faces. Recall that a velocity vector in $\mathbb{R}^{d i m}$ can be recovered from dim orthogonal projections on any dim linearly independent vectors. For example, for a convex non-degenerate cell in $\mathbb{R}^{3}$, any triplet of normal vectors to faces with a common point satisfy the above requirement. These orthogonal projections are chosen as degrees of freedom. The recovered velocities are used to define scalar product (2.2). We now consider two examples.
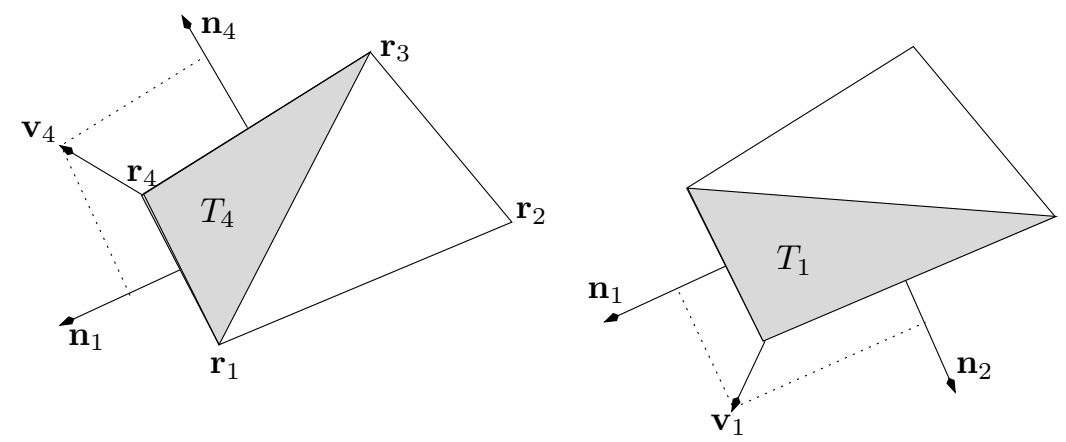

Figure 1: Recovered vectors $\mathbf{v}_{1}, \mathbf{v}_{4}$ and triangles $T_{1}, T_{4}$.

Let $E$ be a convex polygon with $s$ edges ( $s=3$ for a triangle and $s=4$ for a quadrilateral). As illustrated in Fig. 1, four recovered velocity vectors can be associated with the four vertices of a quadrilateral. For example, velocity $\mathbf{v}_{1}$ is recovered from its projections onto the normal vectors $\mathbf{n}_{1}$ and $\mathbf{n}_{2}$. In the general case, we denote by $\mathbf{v}\left(\mathbf{r}_{k}\right)$ the velocity recovered at the $k$-th vertex $\mathbf{r}_{k}$ of $E, k=1, \ldots, s$. In this paper, we shall consider two cell-based scalar products. The first one is given by

$$
[\vec{u}, \vec{v}]_{X_{i}^{d}, E}=\frac{1}{\alpha_{E}} \sum_{k=1}^{s}\left|T_{k}\right| K^{-1}\left(\mathbf{r}_{k}\right) \mathbf{u}\left(\mathbf{r}_{k}\right) \cdot \mathbf{v}\left(\mathbf{r}_{k}\right), \quad \alpha_{E}=\frac{1}{|E|} \sum_{k=1}^{s}\left|T_{k}\right|,
$$

where $\left|T_{k}\right|$ is the area of the triangle formed by two edges sharing the $k$-th vertex. See, for example, the shaded triangles $T_{1}$ and $T_{4}$ in Fig. 1 . The second cell-based scalar product requires only one evaluation of the tensor $K$ and is given by

$$
[\vec{u}, \vec{v}]_{X_{i}^{d}, E}=\frac{1}{\alpha_{E}} \sum_{k=1}^{s}\left|T_{k}\right| K_{E}^{-1} \mathbf{u}\left(\mathbf{r}_{k}\right) \cdot \mathbf{v}\left(\mathbf{r}_{k}\right)
$$

where $K_{E}$ is the value of tensor $K$ at the mass center of $E$.

Note that both (2.2), (2.3) and (2.2), (2.4) are indeed scalar products on $X_{i}^{d}$, since $K$ is a uniformly bounded, symmetric and positive definite tensor, and

$$
c_{1}|E| \sum_{f \subset \partial E} v_{f}^{2} \leq[\vec{v}, \vec{v}]_{X_{i}^{d}, E} \leq c_{2}|E| \sum_{f \subset \partial E} v_{f}^{2}
$$

where $v_{f}$ denotes the velocity component associated with face $f$, and $c_{1}, c_{2}$ are positive constants independent of $h$.

Let $E$ be a convex polyhedron with flat faces such that each vertex of $E$ is shared by exactly three faces. This allows us to uniquely recover velocity vectors at the vertices of $E$. The scalar product over polyhedron $E$ is given either by (2.3) or by (2.4), where $T_{k}$ is the 
tetrahedron formed by the three edges sharing the $k$-th vertex of $E$. Note that for a tetrahedron, $s=4$ and $\alpha_{E}=4$.

The third step in the MFD method is to derive a discrete approximation to the divergence operator, $\mathcal{D} I V^{d}$, which we shall refer to as the prime operator. The divergence theorem gives

$$
\left.\left(\mathcal{D} I V^{d} \vec{u}\right)\right|_{E}=\frac{1}{|E|} \sum_{f \subset \partial E} u_{f}|f|
$$

where $u_{f}$ is the normal velocity components on face $f$ and $|f|$ is the face area. Formula (2.6) assumes an external orientation of normal vectors. If the vector normal to face $f$ points into the element, $u_{f}$ must be replaced by $-u_{f}$. The extended discrete divergence operator, $\mathcal{D}^{d}: X_{i}^{d} \rightarrow Q_{i}^{d}$, is given by

$$
\mathcal{D}^{d} \vec{u}= \begin{cases}\left.\left(\mathcal{D} I V^{d} \vec{u}\right)\right|_{E} & \forall E \in \mathcal{T}_{h, i}, \\ -u_{f} & \forall f \in \mathcal{E}_{h, i} .\end{cases}
$$

In the fourth step of the MFD method, a discrete flux operator $\mathcal{G}^{d}$ that is adjoint to the discrete extended divergence operator $\mathcal{D}^{d}$ with respect to scalar products (2.1) and (2.2) is derived, i.e.

$$
\left[\mathcal{D}^{d} \vec{u}, \vec{p}\right]_{Q_{i}^{d}} \equiv\left[\vec{u}, \mathcal{G}^{d} \vec{p}\right]_{X_{i}^{d}}, \quad \forall \vec{u} \in X_{i}^{d}, \quad \forall \vec{p} \in Q_{i}^{d} .
$$

We will refer to (2.8) as the discrete Green's formula. For the explicit formula of the operator $\mathcal{G}^{d}$, see [5]. Now, the MFD method for subdomain $\Omega_{i}$ may be summarized as follows:

$$
\begin{aligned}
\vec{u} & =\mathcal{G}^{d} \vec{p}, \\
\mathcal{D} I V^{d} \vec{u} & =\vec{b}_{i},
\end{aligned}
$$

where $\vec{b}_{i}$ is in $Q_{i}^{d}$. The entries of $\vec{b}_{i}$ are integral averages of the right-hand side $b$ over the elements of $\mathcal{T}_{h, i}$.

\subsection{Interface conditions}

The system (2.9) is closed by imposing continuity and boundary conditions. Let us consider the interface $\Gamma_{i, j}$ between subdomains $\Omega_{i}$ and $\Omega_{j}$. Hereafter, we will use subscript ' $i$ ' for the vectors and operators satisfying equation (2.9). The continuity conditions that the true solution satisfies are

$$
\left.p_{i}\right|_{\Gamma_{i, j}}=\left.p_{j}\right|_{\Gamma_{i, j}} \quad \text { and }\left.\quad \mathbf{u}_{i} \cdot \mathbf{n}_{i}\right|_{\Gamma_{i, j}}=-\left.\mathbf{u}_{j} \cdot \mathbf{n}_{j}\right|_{\Gamma_{i, j}} .
$$

In order to impose the above conditions discretely on non-matching grids, we introduce the intermediate vector space $\Lambda_{i, j}^{d} \equiv \Lambda_{j, i}^{d}$ associated with the interface partition $\tilde{\mathcal{E}}_{h, i, j}$. We will make precise the definition of $\Lambda_{i, j}^{d}$ later in Section 4 , where it will be related to a mortar space in mixed finite element method.

Denote by $Q_{i, j}^{d}$ the vector space of pressure unknowns associated with the faces of partition $\mathcal{E}_{h, i, j}$. The scalar product in $Q_{i, j}^{d}$ is given by

$$
\left[\vec{p}_{i, j}, \vec{q}_{i, j}\right]_{Q_{i, j}^{d}}=\sum_{f \subset \mathcal{E}_{h, i, j}}|f| p_{i, j, f} q_{i, j, f}
$$

where $p_{i, j, f}$ (resp., $q_{i, j, f}$ ) is the component of vector $\vec{p}_{i, j}$ (resp., $\vec{q}_{i, j}$ ) associated with face $f$. 
Similarly, we define the vector space $X_{i, j}^{d}$ of velocity unknowns associated with the faces of partition $\mathcal{E}_{h, i, j}$. We choose $X_{i, j}^{d}$ to be isometric to $Q_{i, j}^{d}$, i.e.

$$
\left[\vec{u}_{i, j}, \vec{v}_{i, j}\right]_{X_{i, j}^{d}}=\left[\vec{u}_{i, j}, \vec{v}_{i, j}\right]_{Q_{i, j}^{d}} .
$$

Finally, let $R_{i, j}: \Lambda_{i, j}^{d} \rightarrow Q_{i, j}^{d}$ be a linear projection operator exact for constant vectors. We will make precise the definition of $R_{i, j}$ later in Section 4 , where it will be related to the orthogonal projector from the mortar finite element space to the space of piecewise constant functions.

The continuity conditions are derived from two requirements. First, we require the local mass conservation across each face of $\tilde{\mathcal{E}}_{h, i, j}$, i.e.

$$
\left[\vec{u}_{i, j}, R_{i, j} \vec{\mu}\right]_{X_{i, j}^{d}}=-\left[\vec{u}_{j, i}, R_{j, i} \vec{\mu}\right]_{X_{j, i}^{d}} \quad \forall \vec{\mu} \in \Lambda_{i, j}^{d} .
$$

Let $F_{i, j}$ be the diagonal matrix with entries that are the areas of faces of $\mathcal{E}_{h, i, j}$. It is not difficult to see that the mass conservation results in the following interface condition:

$$
R_{i, j}^{T} F_{i, j} \vec{u}_{i, j}=-R_{j, i}^{T} F_{j, i} \vec{u}_{j, i} .
$$

Second, we require that the discrete Green's formula (2.8) holds on $\bar{\Omega}_{i} \cup \bar{\Omega}_{j}$. It is not difficult to see that this will be true if the sum of the two discrete Green's formulas leaves only outer boundary contributions. According to (2.1), the boundary terms associated with the common interface will cancel if

$$
\left[\vec{p}_{i, j}, \vec{u}_{i, j}\right]_{Q_{i, j}^{d}}=-\left[\vec{p}_{j, i}, \vec{u}_{j, i}\right]_{Q_{j, i}^{d}} .
$$

The sufficient condition for that is as follows:

$$
\exists \vec{\lambda} \in \Lambda_{i, j}^{d}: \quad \vec{p}_{i, j}=R_{i, j} \vec{\lambda} \quad \text { and } \quad \vec{p}_{j, i}=R_{j, i} \vec{\lambda}
$$

In a very special case of non-matching meshes, the locally refined meshes, vector $\vec{\lambda}$ can be eliminated from (2.13). In Section 7, we derive simple formulas for the interface conditions on locally refined meshes. In a more general case, $\vec{\lambda}$ is considered as an additional unknown.

The system of equations (2.9) is closed by imposing continuity conditions (2.12) and (2.13) and the boundary conditions

$$
p_{f}=g_{f} \quad \forall f \subset \partial \Omega,
$$

where $g_{f}$ is the integral average of $g$ over face $f$.

\section{Mortar mixed finite element method}

In this section we briefly recall the mortar mixed finite element method introduced in $[23,1]$, which will be later related to the mortar MFD method from the previous section. We shall follow the standard notations for norms, seminorm and scalar products. A weak solution of (1.1)-(1.3) is a pair $\mathbf{u} \in H(\operatorname{div} ; \Omega), p \in L^{2}(\Omega)$, such that

$$
\begin{array}{ll}
\left(K^{-1} \mathbf{u}, \mathbf{v}\right)=(p, \nabla \cdot \mathbf{v})-\langle g, \mathbf{v} \cdot \mathbf{n}\rangle_{\partial \Omega}, & \mathbf{v} \in H(\operatorname{div} ; \Omega), \\
(\nabla \cdot \mathbf{u}, w)=(b, w), & w \in L^{2}(\Omega) .
\end{array}
$$


It is well known (see, e.g., $[7,18])$ that $(3.1)-(3.2)$ has an unique solution. The multidomain formulation of (3.1)-(3.1) is based on the spaces

$$
\begin{array}{ll}
\mathbf{V}_{i}=H\left(\operatorname{div} ; \Omega_{i}\right), & \mathbf{V}=\bigoplus_{i=1}^{n} \mathbf{V}_{i}, \\
W_{i}=L^{2}\left(\Omega_{i}\right), & W=\bigoplus_{i=1}^{n} W_{i}=L^{2}(\Omega) .
\end{array}
$$

If the solution $(\mathbf{u}, p)$ of (3.1)-(3.2) belongs to $H(\operatorname{div} ; \Omega) \times H^{1}(\Omega)$, it is easy to see [7, pp. 9192] that it satisfies, for $1 \leq i \leq n$,

$$
\begin{aligned}
\left(K^{-1} \mathbf{u}, \mathbf{v}\right)_{\Omega_{i}} & =(p, \nabla \cdot \mathbf{v})_{\Omega_{i}}-\left\langle p, \mathbf{v} \cdot \mathbf{n}_{i}\right\rangle_{\Gamma_{i}}-\left\langle g, \mathbf{v} \cdot \mathbf{n}_{i}\right\rangle_{\partial \Omega_{i} \backslash \Gamma}, & \mathbf{v} & \in \mathbf{V}_{i}, \\
(\nabla \cdot \mathbf{u}, w)_{\Omega_{i}} & =(b, w)_{\Omega_{i}}, & w & \in W_{i} .
\end{aligned}
$$

The mortar mixed finite element method discretizes (3.3)-(3.4), coupled with a mortar-based discretization of the continuity conditions (2.10). Next, we present the definition of the mixed finite element spaces. We restrict ourself to the two-dimensional elements: quadrilaterals and triangles. The finite element spaces for a tetrahedral element are constructed similar to the finite element spaces for a triangular element.

For any element $E \in \mathcal{T}_{h}$, there exists a bijection mapping $F_{E}: \hat{E} \rightarrow E$, where $\hat{E}$ is the reference element. For example, in the case of convex quadrilaterals, $\hat{E}$ is the unit square with vertices $\hat{\mathbf{r}}_{1}=(0,0)^{T}, \hat{\mathbf{r}}_{2}=(1,0)^{T}, \hat{\mathbf{r}}_{3}=(1,1)^{T}$ and $\hat{\mathbf{r}}_{4}=(0,1)^{T}$. Denote by $\mathbf{r}_{i}=\left(x_{i}, y_{i}\right)^{T}, i=1,2,3,4$, the four corresponding vertices of element $E$ as shown in Fig. 2. Then, $F_{E}$ is the bilinear mapping given by

$$
F_{E}(\hat{\mathbf{r}})=\mathbf{r}_{1}(1-\hat{x})(1-\hat{y})+\mathbf{r}_{2} \hat{x}(1-\hat{y})+\mathbf{r}_{3} \hat{x} \hat{y}+\mathbf{r}_{4}(1-\hat{x}) \hat{y} .
$$

Note that the Jacobi matrix $D F_{E}$ and its Jacobian $J_{E}$ are linear functions of $\hat{x}$ and $\hat{y}$. Indeed, straightforward computations yield

$$
D F_{E}=\left[(1-\hat{y}) \mathbf{r}_{21}+\hat{y} \mathbf{r}_{34},(1-\hat{x}) \mathbf{r}_{41}+\hat{x} \mathbf{r}_{32}\right],
$$

and

$$
J_{E}=2\left|T_{124}\right|+2\left(\left|T_{123}\right|-\left|T_{124}\right|\right) \hat{x}+2\left(\left|T_{134}\right|-\left|T_{124}\right|\right) \hat{y}
$$

where $\mathbf{r}_{i j}=\mathbf{r}_{i}-\mathbf{r}_{j}$ and $\left|T_{i j k}\right|$ is the area of the triangle with vertices $\mathbf{r}_{i}, \mathbf{r}_{j}$ and $\mathbf{r}_{k}$. Since $E$ is convex, the Jacobian $J_{E}$ is uniformly positive, i.e. $J_{E}(\hat{x}, \hat{y})>0$. We denote the inverse mapping by $F_{E}^{-1}$ and its Jacobian by $J_{F_{E}^{-1}}$.

In the case of triangles, $\hat{E}$ is the reference right triangle with vertices $\hat{\mathbf{r}}_{1}=(0,0)^{T}$, $\hat{\mathbf{r}}_{2}=(1,0)^{T}$, and $\hat{\mathbf{r}}_{3}=(0,1)^{T}$. The linear mapping for triangles has the form

$$
F_{E}(\hat{\mathbf{r}})=\mathbf{r}_{1}(1-\hat{x}-\hat{y})+\mathbf{r}_{2} \hat{x}+\mathbf{r}_{3} \hat{y}
$$

with respective Jacobi matrix and Jacobian

$$
D F_{E}=\left[\mathbf{r}_{21}, \mathbf{r}_{31}\right]^{T} \quad \text { and } \quad J_{E}=2\left|T_{123}\right| .
$$

Note that in this case the mapping is affine and the Jacobi matrix and its Jacobian are constants.

We denote the lowest order Raviart-Thomas-Nedelec (RTN) mixed finite element spaces $[20,17,16]$ by

$$
\mathbf{V}_{h, i} \times W_{h, i} \subset \mathbf{V}_{i} \times W_{i}
$$




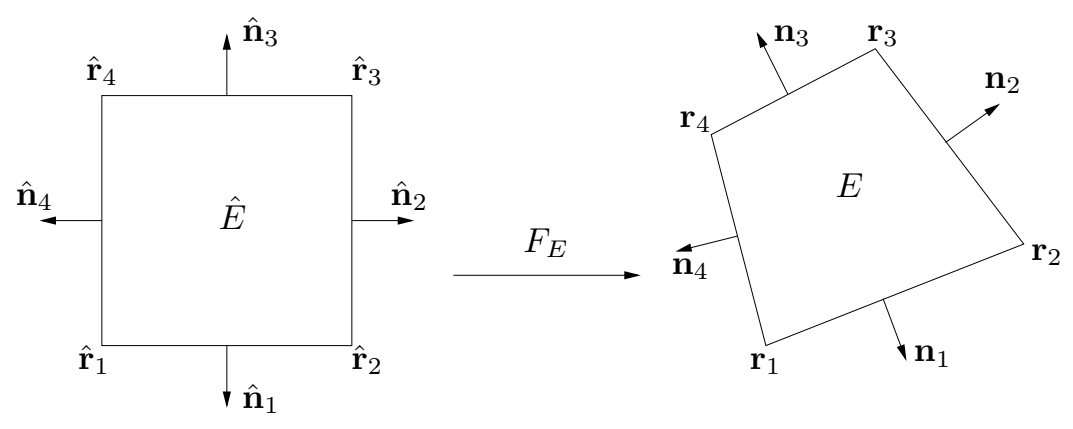

Figure 2: Bilinear mapping and orientation of normal vectors.

These spaces are initially defined on the reference element. For example, if $\hat{E}$ is the unit square, the spaces are

$$
\hat{\mathbf{V}}(\hat{E})=P_{1,0}(\hat{E}) \times P_{0,1}(\hat{E}) \quad \text { and } \quad \hat{W}(\hat{E})=P_{0}(\hat{E}),
$$

where $P_{1,0}$ (or $P_{0,1}$ ) denotes the space of polynomials linear in the $\hat{x}$ (or $\hat{y}$ ) variable and constant in the other variable, and $P_{0}$ denotes the space of constant functions. In the case when $\hat{E}$ is the unit triangle, the spaces on this reference element are

$$
\hat{\mathbf{V}}(\hat{E})=P_{0}(\hat{E}) \times P_{0}(\hat{E})+P_{0}(\hat{E}) \hat{\mathbf{x}} \quad \text { and } \quad \hat{W}(\hat{E})=P_{0}(\hat{E}) .
$$

The velocity space on any element $E$ is defined via the Piola transformation

$$
\frac{1}{J_{E}} D F_{E}:\left(L_{2}(\hat{E})\right)^{d i m} \rightarrow\left(L_{2}(E)\right)^{d i m}, \quad \forall E \in \mathcal{T}_{h}
$$

The RTN $N_{0}$ spaces on $\mathcal{T}_{h, i}$ are given by

$$
\begin{aligned}
\mathbf{V}_{h, i}=\left\{\mathbf{v} \in \mathbf{V}_{i}:\right. & \left.\left.\mathbf{v}\right|_{E}=J_{E}^{-1} D F_{E} \hat{\mathbf{v}} \circ F_{E}^{-1}, \hat{\mathbf{v}} \in \hat{\mathbf{V}}(\hat{E}) \quad \forall E \in \mathcal{T}_{h, i}\right\}, \\
W_{h, i}=\left\{w \in W_{i}:\right. & \left.\left.w\right|_{E}=\hat{w} \circ F_{E}^{-1}, \hat{w} \in \hat{W}(\hat{E}) \quad \forall E \in \mathcal{T}_{h, i}\right\} .
\end{aligned}
$$

The following two properties of the Piola transformation will be useful in the analysis. For any $\hat{\mathbf{v}} \in \hat{\mathbf{V}}(\hat{E})$ and the related $\mathbf{v}=J_{E}^{-1} D F_{E} \hat{\mathbf{v}} \circ F_{E}^{-1}$, we have

$$
\int_{E} \nabla \cdot \mathbf{v} \mathrm{d} \mathbf{x}=\int_{\hat{E}} \hat{\nabla} \cdot \hat{\mathbf{v}} \mathrm{d} \hat{\mathbf{x}} \quad \text { and } \quad \int_{f} \mathbf{v} \cdot \mathbf{n}_{f} \mathrm{~d} s=\int_{\hat{f}} \hat{\mathbf{v}} \cdot \hat{\mathbf{n}}_{\hat{f}} \mathrm{~d} \hat{s},
$$

where $f$ is any face of $E$ and $\mathbf{n}_{f}$ and $\hat{\mathbf{n}}_{\hat{f}}$ are the unit normal vectors to $f$ and $\hat{f}$, respectively.

The quasi-uniform partition $\tilde{\mathcal{E}}_{h, i, j}$ of $\Gamma_{i, j}$ introduced above is referred to as the mortar interface mesh. Denote by $\Lambda_{h, i, j} \subset L^{2}\left(\Gamma_{i, j}\right)$ the mortar space on $\Gamma_{i, j}$, containing either the continuous or discontinuous piecewise polynomials of degree one on $\tilde{\mathcal{E}}_{h, i, j}$. Let

$$
\mathbf{V}_{h}=\bigoplus_{i=1}^{n} \mathbf{V}_{h, i}, \quad W_{h}=\bigoplus_{i=1}^{n} W_{h, i}, \quad \Lambda_{h}=\bigoplus_{1 \leq i<j \leq n} \Lambda_{h, i, j} .
$$

Although normal components of vectors in $\mathbf{V}_{h}$ are continuous between elements within each block $\Omega_{i}$, there is no such restriction across $\Gamma$. The space $\Lambda_{h}$ is called the mortar finite element space on $\Gamma$. In the following we treat any function $\mu \in \Lambda_{h}$ as extended by zero on $\partial \Omega$. An 
additional assumption on the space $\Lambda_{h}$ and, hence, $\tilde{\mathcal{E}}_{h, i, j}$ will be made below in (4.7) and (5.13). We remark that $\tilde{\mathcal{E}}_{h, i, j}$ need not be conforming if a discontinuous space is used.

In the mortar mixed finite element approximation of (3.1)-(3.2), we seek $\mathbf{u}_{h} \in \mathbf{V}_{h}, p_{h} \in$ $W_{h}, \lambda_{h} \in \Lambda_{h}$ such that, for $1 \leq i \leq n$,

$$
\begin{array}{rlrl}
\left(K^{-1} \mathbf{u}_{h}, \mathbf{v}\right)_{\Omega_{i}} & =\left(p_{h}, \nabla \cdot \mathbf{v}\right)_{\Omega_{i}}-\left\langle\lambda_{h}, \mathbf{v} \cdot \mathbf{n}_{i}\right\rangle_{\Gamma_{i}}-\left\langle g, \mathbf{v} \cdot \mathbf{n}_{i}\right\rangle_{\partial \Omega_{i} \backslash \Gamma}, & \mathbf{v} \in \mathbf{V}_{h, i} \\
\left(\nabla \cdot \mathbf{u}_{h}, w\right)_{\Omega_{i}} & =(b, w)_{\Omega_{i}}, & w & \in W_{h, i}, \\
\sum_{i=1}^{n}\left\langle\mathbf{u}_{h} \cdot \mathbf{n}_{i}, \mu\right\rangle_{\Gamma_{i}} & =0, & \mu \in \Lambda_{h} .
\end{array}
$$

Remark 3.1 The above method imposes continuity of pressure by approximating the pressure on the interfaces by a single-valued mortar function $\lambda_{h}$, while continuity of normal flux is imposed weakly in (3.12) with respect to Lagrange multipliers in the mortar space.

\section{Relating mortar MFD and mortar MFE methods}

The basic tool for the error analysis of the mortar MFD method is based on establishing connections with the mortar mixed finite element (MFE) method (3.10)-(3.12). We begin by establishing an isomorphism between finite difference and finite element spaces.

The degrees of freedom of $\mathbf{V}_{h, i}$ are associated with mesh faces. Therefore, the space $\mathbf{V}_{h, i}$ is isomorphic to the vector space $X_{i}^{d}$. Similarly, the degrees of freedom of the finite element space $W_{h, i}$ are associated with the cell centers and the space is isometric to the vector space $Q_{i}^{d, 0}$ (see [5]). By the same arguments, the vector space $X_{i, j}^{d}$ is isometric to the finite element space $\left.\mathbf{V}_{h, i} \cdot \mathbf{n}_{i}\right|_{\Gamma_{i, j}}$.

Finally, we chose $\Lambda_{i, j}^{d}$ to be isomorphic to finite element space $\Lambda_{h, i, j}$. In particular, the degrees of freedom of $\Lambda_{i, j}^{d}$ are the values of the pressure at the vertices of partition $\tilde{\mathcal{E}}_{h, i, j}$. In the case of discontinuous mortars, each vertex may be associated with multiple degrees of freedom. The projector $R_{i, j}$ is implicitly given by

$$
\left[R_{i, j} \vec{\mu}_{i, j}, \vec{q}_{i, j}\right]_{Q_{i, j}^{d}}=\left\langle\mu_{h, i, j}, q_{h, i, j}\right\rangle_{\Gamma_{i, j}}, \quad \forall \vec{\mu}_{i, j} \in \Lambda_{i, j}^{d}, \forall \vec{q}_{i, j} \in Q_{i, j}^{d},
$$

where $\mu_{h, i, j} \in \Lambda_{h, i, j}$ and $\left.q_{h, i, j} \in \mathbf{V}_{h, i} \cdot \mathbf{n}_{i}\right|_{\Gamma_{i, j}}$ are the finite element counterparts of vectors $\vec{\mu}_{i, j}$ and $\vec{q}_{i, j}$, respectively.

For each interface $\Gamma_{i, j}$, we define an $L^{2}$-orthogonal projection operator $\mathcal{R}_{h, i, j}: L^{2}\left(\Gamma_{i, j}\right) \rightarrow$ $\left.\mathbf{V}_{h, i} \cdot \mathbf{n}_{i}\right|_{\Gamma_{i, j}}$ such that, for any $\phi \in L^{2}\left(\Gamma_{i, j}\right)$,

$$
\left\langle\phi-\mathcal{R}_{h, i, j} \phi, \mathbf{v} \cdot \mathbf{n}_{i}\right\rangle_{\Gamma_{i, j}}=0 \quad \forall \mathbf{v} \in \mathbf{V}_{h, i} .
$$

The operator $\mathcal{R}_{h, j, i}:\left.L^{2}\left(\Gamma_{i, j}\right) \rightarrow \mathbf{V}_{h, j} \cdot \mathbf{n}_{j}\right|_{\Gamma_{i, j}}$ is defined similarly. Let $\mathcal{R}_{h, i}: L^{2}\left(\partial \Omega_{i}\right) \rightarrow$ $\left.\mathbf{V}_{h, i} \cdot \mathbf{n}_{i}\right|_{\partial \Omega_{i}}$ be such that, for any $\phi \in L^{2}\left(\partial \Omega_{i}\right)$,

$$
\left.\mathcal{R}_{h, i} \phi\right|_{\Gamma_{i, j}}=\mathcal{R}_{h, i, j} \phi
$$

Note that the projection operator $\mathcal{R}_{h, i, j}$ restricted to $\Lambda_{h, i, j}$ acts from the space of piecewise linear functions on $\tilde{\mathcal{E}}_{h, i, j}$ to the space of piecewise constant functions on $\mathcal{E}_{h, i, j}$. Using (4.1), it is clear that the projector $R_{i, j}$ defined on the vector space $\Lambda_{i, j}^{d}$ is the matrix representation of $\mathcal{R}_{h, i, j}:\left.\Lambda_{h, i, j} \rightarrow \mathbf{V}_{h, i} \cdot \mathbf{n}_{i}\right|_{\Gamma_{i, j}}$. 
The next step is to reformulate the MFD method in a way that is more suitable for our analysis. Multiplying the first equation in (2.9) by $\vec{v}_{i} \in X_{i}^{d}$, the second one by $\vec{q}_{i} \in Q_{i}^{d, 0}$, and using the discrete Green's formula (2.8), we get

$$
\begin{array}{lll}
{\left[\vec{u}_{i}, \vec{v}_{i}\right]_{X_{i}^{d}}-\left[\vec{p}_{i}, \mathcal{D}_{i}^{d} \vec{v}_{i}\right]_{Q_{i}^{d}}} & =0, & \vec{v}_{i} \in X_{i}^{d}, \\
{\left[\vec{q}_{i}, \mathcal{D} I V_{i}^{d} \vec{u}_{i}\right]_{Q_{i}^{d, 0}}} & =\left[\vec{b}_{i}, \vec{q}_{i}\right]_{Q_{i}^{d, 0},} & \vec{q}_{i} \in Q_{i}^{d, 0} .
\end{array}
$$

Recall that the above equations are coupled with the continuity conditions (2.12), (2.13) and the boundary conditions (2.14). Using the isomorphism between the finite element space $\mathbf{V}_{h, i} \times W_{h, i}$ and the vector space $X_{i}^{d} \times Q_{i}^{d, 0}$, we define finite element functions $q_{h, i}, b_{h, i}$ and $\mathbf{u}_{h, i}$ corresponding to vectors $\vec{q}_{i}, \vec{b}_{i}$ and $\vec{u}_{i}$, respectively. Then,

$$
\left[\vec{q}_{i}, \mathcal{D} I V_{i}^{d} \vec{u}_{i}\right]_{Q_{i}^{d, 0}}=\left(q_{h, i}, \nabla \cdot \mathbf{u}_{h, i}\right)_{\Omega_{i}} .
$$

The definition of $\vec{b}_{i}$ implies that

$$
\left[\vec{b}_{i}, \vec{q}_{i}\right]_{Q_{i}^{d, 0}}=\left(b_{h, i}, q_{h, i}\right)_{\Omega_{i}}=\left(b, q_{h, i}\right)_{\Omega_{i}} .
$$

We decompose vector $\vec{p}_{i}$ as $\vec{p}_{i}=\left(\vec{p}_{i}^{0}, \vec{p}_{i}^{1}\right)$, where $\vec{p}_{i}^{0} \in Q_{i}^{d, 0}$, and denote the finite element counterparts of $\vec{p}_{i}^{0}$ and $\vec{v}_{i} \in X_{i}^{d}$ by $p_{h, i}$ and $\mathbf{v}_{h, i}$, respectively. Let $\lambda_{h} \in \Lambda_{h}$ be the mortar finite element counterpart of $\vec{\lambda}$ from the pressure continuity condition (2.13). The Dirichlet boundary conditions specify the components of vector $\vec{p}_{i}^{1}$ on $\partial \Omega$. Using (2.7), (2.13), (2.14), and the definition of the projectors $R_{i, j}$ and $\mathcal{R}_{h, i}$, we get

$$
\begin{aligned}
{\left[\vec{p}_{i}, \mathcal{D}_{i}^{d} \vec{v}_{i}\right]_{Q_{i}^{d}} } & =\left(p_{h, i}, \nabla \cdot \mathbf{v}_{h, i}\right)_{\Omega_{i}}-\left\langle\mathcal{R}_{h, i} \lambda_{h}, \mathbf{v}_{h, i} \cdot \mathbf{n}_{i}\right\rangle_{\Gamma_{i}}-\left\langle\mathcal{R}_{h, i} g, \mathbf{v}_{h, i} \cdot \mathbf{n}_{i}\right\rangle_{\partial \Omega_{i} \backslash \Gamma} \\
& =\left(p_{h, i}, \nabla \cdot \mathbf{v}_{h, i}\right)_{\Omega_{i}}-\left\langle\lambda_{h}, \mathbf{v}_{h, i} \cdot \mathbf{n}_{i}\right\rangle_{\Gamma_{i}}-\left\langle g, \mathbf{v}_{h, i} \cdot \mathbf{n}_{i}\right\rangle_{\partial \Omega_{i} \backslash \Gamma} .
\end{aligned}
$$

Next, letting $\mu_{h, i, j} \in \Lambda_{h, i, j}$ be the finite element counterpart of vector $\vec{\mu}_{i, j}$, the continuity condition (2.11) becomes

$$
\left\langle\mu_{h, i, j}, \mathbf{u}_{h, i} \cdot \mathbf{n}_{i}\right\rangle_{\Gamma_{i, j}}=-\left\langle\mu_{h, i, j}, \mathbf{u}_{h, j} \cdot \mathbf{n}_{j}\right\rangle_{\Gamma_{j, i}} .
$$

Finally, by introducing the quadrature rule

$$
\left(K^{-1} \mathbf{u}_{h, i}, \mathbf{v}_{h, i}\right)_{h, \Omega_{i}} \equiv\left[\vec{u}_{i}, \vec{v}_{i}\right]_{X_{i}^{d}}
$$

we can reformulate the mortar MFD problem (2.9), (2.13), (2.12), (2.14) as the following problem. We seek $\mathbf{u}_{h} \in \mathbf{V}_{h}, p_{h} \in W_{h}, \lambda_{h} \in \Lambda_{h}$ such that, for $1 \leq i \leq n$,

$$
\begin{array}{rlrl}
\left(K^{-1} \mathbf{u}_{h}, \mathbf{v}\right)_{h, \Omega_{i}} & =\left(p_{h}, \nabla \cdot \mathbf{v}\right)_{\Omega_{i}}-\left\langle\lambda_{h}, \mathbf{v} \cdot \mathbf{n}_{i}\right\rangle_{\Gamma_{i}}-\left\langle g, \mathbf{v} \cdot \mathbf{n}_{i}\right\rangle_{\partial \Omega_{i} \backslash \Gamma}, & \mathbf{v} & \in \mathbf{V}_{h, i} \\
\left(\nabla \cdot \mathbf{u}_{h}, w\right)_{\Omega_{i}} & =(b, w)_{\Omega_{i}}, & w & \in W_{h, i} \\
\sum_{i=1}^{n}\left\langle\mathbf{u}_{h} \cdot \mathbf{n}_{i}, \mu\right\rangle_{\Gamma_{i}} & =0, & \mu \in \Lambda_{h} .
\end{array}
$$

The next lemma shows that the problem is well posed.

Lemma 4.1 Assume that for any $\phi \in \Lambda_{h}$,

$$
\mathcal{R}_{h, i} \phi=0,1 \leq i \leq n \text {, implies that } \phi=0 .
$$

Then there exists a unique solution of (4.4)-(4.6). 
Proof. The proof closely follows the proof of Lemma 2.1 in [1] with only a slight modification. Since (4.4)-(4.6) is a square system, it is sufficient to show uniqueness. Let $b=0$, $g=0$. Setting $\mathbf{v}=\mathbf{u}_{h}, w=p_{h}$, and $\mu=-\lambda_{h}$, adding (4.4)-(4.6), and summing over $1 \leq i \leq n$, implies that

$$
\sum_{i=1}^{n}\left(K^{-1} \mathbf{u}_{h}, \mathbf{u}_{h}\right)_{h, \Omega_{i}}=0 .
$$

The coercivity result from [5]

$$
\sum_{i=1}^{n}\left(K^{-1} \mathbf{u}_{h}, \mathbf{u}_{h}\right)_{h, \Omega_{i}} \geq C\left\|\mathbf{u}_{h}\right\|_{0, \Omega}^{2}
$$

implies that $\mathbf{u}_{h}=0$. The argument for proving that $p_{h}=\lambda_{h}=0$ is the same as in the proof of Lemma 2.1 in [1].

Remark 4.1 Above, as well as in several other places in this paper, we employ results obtained in [1]. Although [1] only treats affine elements, it is easy to check that the arguments used to obtain the results referred to here also apply in the case of general quadrilateral elements.

We end this section by remarking that (4.8) and the continuity of scalar products $(\cdot, \cdot)_{h, \Omega_{i}}$ imply that these scalar products give rise to a norm $\|\cdot\|_{h}$ in $\mathbf{V}_{h}$, which is equivalent to the $L^{2}$-norm. In other words, there exist positive constants $c_{1}$ and $c_{2}$ independent of $h$, such that

$$
c_{1}\|\mathbf{v}\|_{0, \Omega} \leq\|\mathbf{v}\|_{h} \leq c_{2}\|\mathbf{v}\|_{0, \Omega} \quad \forall \mathbf{v} \in \mathbf{V}_{h} .
$$

\section{Velocity error estimates}

We first recall several projection operators that will be used in the analysis. On each $\Omega_{i}$ there exists a projection $\Pi_{i}$ from $\left(H^{1}\left(\Omega_{i}\right)\right)^{\text {dim }}$ onto $\mathbf{V}_{h, i}$ satisfying

$$
\left(\nabla \cdot\left(\Pi_{i} \mathbf{q}-\mathbf{q}\right), w\right)_{\Omega_{i}}=0, \quad w \in W_{h, i} .
$$

Let $\Pi: \bigoplus_{i=1}^{n}\left(H^{1}\left(\Omega_{i}\right)\right)^{\operatorname{dim}} \rightarrow \mathbf{V}_{h}$ be defined by $\left.(\Pi \mathbf{q})\right|_{\Omega_{i}}=\Pi_{i}\left(\left.\mathbf{q}\right|_{\Omega_{i}}\right)$. The operator $\Pi$ is defined locally on each element $E$ by

$$
\widehat{\Pi \mathbf{q}}=\hat{\Pi} \hat{\mathbf{q}}
$$

where $\hat{\Pi}:\left(H^{1}(\hat{E})\right)^{\operatorname{dim}} \rightarrow \hat{\mathbf{V}}(\hat{E})$ is the reference element projection operator satisfying

$$
\int_{\hat{f}}(\hat{\Pi} \hat{\mathbf{q}}-\hat{\mathbf{q}}) \cdot \hat{\mathbf{n}}=0 \quad \forall \hat{f} \subset \partial \hat{E} .
$$

Let $\mathcal{P}_{h}$ be the $L^{2}(\Gamma)$ projection onto $\Lambda_{h}$ satisfying for any $\psi \in L^{2}(\Gamma)$,

$$
\left\langle\psi-\mathcal{P}_{h} \psi, \mu\right\rangle_{\Gamma}=0 \quad \forall \mu \in \Lambda_{h}
$$

For any $\varphi \in L^{2}(\Omega)$, let $\mathcal{Q}_{h} \varphi \in W_{h}$ be its $L^{2}(\Omega)$ projection satisfying

$$
\left(\varphi-\mathcal{Q}_{h} \varphi, w\right)_{\Omega}=0 \quad \forall w \in W_{h} .
$$


We state several well-known approximation properties of these projection operators:

$$
\begin{array}{ll}
\left\|\psi-\mathcal{P}_{h} \psi\right\|_{0, \Gamma_{i, j}} \leq C\|\psi\|_{r, \Gamma_{i, j}} h^{r}, & 0 \leq r \leq 2, \\
\left\|\varphi-\mathcal{Q}_{h} \varphi\right\|_{0} \leq C\|\varphi\|_{r, \Omega_{i}} h^{r}, & 0 \leq r \leq 1, \\
\left\|\mathbf{q}-\Pi_{i} \mathbf{q}\right\|_{0, \Omega_{i}} \leq C\|\mathbf{q}\|_{1, \Omega_{i}} h, & \\
\left\|\nabla \cdot\left(\mathbf{q}-\Pi_{i} \mathbf{q}\right)\right\|_{0, \Omega_{i}} \leq C\|\mathbf{q}\|_{r+1, \Omega_{i}} h^{r}, & 0 \leq r \leq 1,
\end{array}
$$

where $\|\cdot\|_{r}$ is the $H^{r}$-norm. Bounds (5.2) and (5.3) are standard $L^{2}$-projection approximation results [9]; bounds (5.4) and (5.5) can be found in [7, 18] for affine elements and [20, 21] for quadrilaterals.

We will also make use of the following continuity bound for $\Pi$.

Lemma 5.1 For all elements $E$ and for all $\mathbf{q} \in\left(H^{1}(E)\right)^{\text {dim }}$, there exists a constant $C$ independent of $h$ such that

$$
\|\Pi \mathbf{q}\|_{1, E} \leq C\|\mathbf{q}\|_{1, E} .
$$

Proof. Let us first consider the case of simplicial grids in two and three dimensions. It is well known [17] that for all $E \in \mathcal{T}_{h}$

$$
\|\Pi \mathbf{q}\|_{H(\operatorname{div} ; E)} \leq C\|\mathbf{q}\|_{1, E}
$$

The definition of $\mathbf{V}_{h}$ on simplices gives that on each $E$, we have $\nabla \cdot \Pi \mathbf{q}=\frac{1}{d i m} \frac{\partial(\Pi \mathbf{q})_{i}}{\partial x_{i}}$, $i=1, \ldots$, dim, which, combined with the above formula, implies the assertion of the lemma.

In the case of quadrilateral grids, it follows from the definition of the bilinear mapping that for all $\hat{\mathbf{x}} \in \hat{E}$ and $s=0,1$

$$
\begin{gathered}
\left|D F_{E}(\hat{\mathbf{x}})\right|_{s, \infty, \hat{E}} \leq C h,\left|J_{E}(\hat{\mathbf{x}})\right|_{s, \infty, \hat{E}} \leq C h^{2},\left|\frac{1}{J_{E}} D F_{E}\right|_{s, \infty, \hat{E}} \leq C h^{-1}, \\
\left|F_{E}^{-1}\right|_{1, \infty, \hat{E}} \leq C h^{-1}, \quad\left\|J_{F_{E}^{-1}}\right\|_{\infty, \hat{E}} \leq C h^{-2}
\end{gathered}
$$

The rest of the proof is based on the inverse inequality which is not a trivial result for a general quadrilateral. For the sake of completeness, we prove it below. The definition (3.8) implies

$$
\int_{E}\left|\frac{\partial \mathbf{q}}{\partial x_{i}}\right|^{2} \mathrm{~d} \mathbf{x}=\int_{\hat{E}}\left|\frac{\partial}{\partial x_{i}}\left(\frac{1}{J_{E}} D F_{E} \hat{\mathbf{q}}\right)\right|^{2}\left|J_{E}\right| \mathrm{d} \hat{\mathbf{x}} .
$$

Thus, using (5.6) and (5.7), we get

$$
\begin{aligned}
|\mathbf{q}|_{1, E} \leq C( & \left\|\frac{1}{J_{E}} D F_{E}\right\|_{\infty, \hat{E}}\left|F_{E}^{-1}\right|_{1, \infty, \hat{E}}\left\|J_{E}\right\|_{\infty, \hat{E}}^{1 / 2}|\hat{\mathbf{q}}|_{1, \hat{E}} \\
& \left.+\left\|J_{E}\right\|_{\infty, \hat{E}}^{1 / 2}\left|\frac{1}{J_{E}} D F_{E}\right|_{1, \infty, \hat{E}}\left|F_{E}^{-1}\right|_{1, \infty, \hat{E}}\|\hat{\mathbf{q}}\|_{0, \hat{E}}\right) \\
\leq C h^{-1}\|\hat{\mathbf{q}}\|_{1, \hat{E}} . &
\end{aligned}
$$

Similarly, we get the estimates

$$
\|\mathbf{q}\|_{0, E} \leq C\|\hat{\mathbf{q}}\|_{0, \hat{E}} \quad \text { and } \quad\|\hat{\mathbf{q}}\|_{0, \hat{E}} \leq C\|\mathbf{q}\|_{0, E}
$$


Combining (5.8) and (5.9) and using the standard inverse inequality on the reference element $\hat{E}$, we get

$$
|\mathbf{q}|_{1, E} \leq C h^{-1}\|\hat{\mathbf{q}}\|_{1, \hat{E}} \leq C h^{-1}\|\hat{\mathbf{q}}\|_{0, \hat{E}} \leq C h^{-1}\|\mathbf{q}\|_{0, E}
$$

Using the inverse inequality, we have

$$
\begin{aligned}
|\Pi \mathbf{q}|_{1, E} & =\left|\Pi \mathbf{q}-\mathbf{q}_{0}\right|_{1, E} \leq C h^{-1}\left\|\Pi \mathbf{q}-\mathbf{q}_{0}\right\|_{0, E} \\
& \leq C h^{-1}\left(\|\Pi \mathbf{q}-\mathbf{q}\|_{0, E}+\left\|\mathbf{q}-\mathbf{q}_{0}\right\|_{0, E}\right)
\end{aligned}
$$

where $\mathbf{q}_{0}$ is a constant vector. Let $\mathbf{q}_{0}$ be the $L^{2}(E)$ projection of $\mathbf{q}$ onto the space of constant vectors. The approximation properties (5.3) and (5.4) result in the estimate

$$
|\Pi \mathbf{q}|_{1, E} \leq C\|\mathbf{q}\|_{1, E} .
$$

The bound $\|\Pi \mathbf{q}\|_{0, E} \leq C\|\mathbf{q}\|_{1, E}$ follows from the approximation property (5.4). This proves the assertion of the lemma.

Throughout the paper we will be using the nonstandard trace theorem [11, Theorem 1.5.2.1]

$$
\|q\|_{r, \Gamma_{i, j}} \leq C\|q\|_{r+1 / 2, \Omega_{i}} .
$$

We will also make use of the trace inequality

$$
\left\|\mathbf{v} \cdot \mathbf{n}_{i}\right\|_{0, \partial \Omega_{i}} \leq C h^{-1 / 2}\|\mathbf{v}\|_{0, \Omega_{i}}, \quad \forall \mathbf{v} \in \mathbf{V}_{h, i},
$$

which follows from a simple scaling argument.

Let

$$
\mathbf{V}_{h, 0}=\left\{\mathbf{v} \in \mathbf{V}_{h}: \sum_{i=1}^{n}\left\langle\left.\mathbf{v}\right|_{\Omega_{i}} \cdot \mathbf{n}_{i}, \mu\right\rangle_{\Gamma_{i}}=0 \quad \forall \mu \in \Lambda_{h}\right\}
$$

be the space of weakly continuous velocities, with respect to the mortar space. Then the mimetic finite difference method (4.4)-(4.6) can be rewritten in the following way. Find $\mathbf{u}_{h} \in \mathbf{V}_{h, 0}$ and $p_{h} \in W_{h}$ such that

$$
\begin{aligned}
\left(K^{-1} \mathbf{u}_{h}, \mathbf{v}\right)_{h} & =\sum_{i=1}^{n}\left(p_{h}, \nabla \cdot \mathbf{v}\right)_{\Omega_{i}}-\langle g, \mathbf{v} \cdot \mathbf{n}\rangle_{\partial \Omega}, \\
\sum_{i=1}^{n}\left(\nabla \cdot \mathbf{u}_{h}, w\right)_{\Omega_{i}} & =(b, w),
\end{aligned}
$$

for $\mathbf{v} \in \mathbf{V}_{h, 0}$ and $w \in W_{h}$. It was shown in [1] that there exists a projection operator $\Pi_{0}$ onto $\mathbf{V}_{h, 0}$ such that, for any $\mathbf{q} \in\left(H^{1}(\Omega)\right)^{d i m}$,

$$
\left(\nabla \cdot\left(\Pi_{0} \mathbf{q}-\mathbf{q}\right), w\right)_{\Omega}=0, \quad w \in W_{h} .
$$

Moreover, if there exists a constant $C$, independent of $h$, such that

$$
\|\mu\|_{0, \Gamma_{i, j}} \leq C\left(\left\|\mathcal{R}_{h, i} \mu\right\|_{0, \Gamma_{i, j}}+\left\|\mathcal{R}_{h, j} \mu\right\|_{0, \Gamma_{i, j}}\right), \forall \mu \in \Lambda_{h}, \quad 1 \leq i<j \leq n,
$$

then $\Pi_{0}$ satisfies the approximation properties

$$
\left\|\Pi_{0} \mathbf{q}-\Pi \mathbf{q}\right\|_{0} \leq C \sum_{i=1}^{n}\|\mathbf{q}\|_{r+1 / 2, \Omega_{i}} h^{r+1 / 2}, \quad 0 \leq r \leq 1,
$$

and

$$
\left\|\Pi_{0} \mathbf{q}-\mathbf{q}\right\|_{0} \leq C \sum_{i=1}^{n}\|\mathbf{q}\|_{1, \Omega_{i}} h
$$




\subsection{Optimal convergence}

In this section we prove optimal error estimates for the mortar MFD method. The analysis is the same for both scalar products (2.3) and (2.4).

Subtracting (5.11)-(5.12) from (3.3)-(3.4) gives the error equations

$$
\begin{aligned}
\left(K^{-1}\left(\Pi \mathbf{u}-\mathbf{u}_{h}\right), \mathbf{v}\right)_{h}= & \sum_{i=1}^{n}\left(\left(p-p_{h}, \nabla \cdot \mathbf{v}\right)_{\Omega_{i}}-\left\langle p, \mathbf{v} \cdot \mathbf{n}_{i}\right\rangle_{\Gamma_{i}}\right) \\
& +\left(K^{-1}(\Pi \mathbf{u}-\mathbf{u}), \mathbf{v}\right)-\sigma\left(K^{-1} \Pi \mathbf{u}, \mathbf{v}\right), \\
\sum_{i=1}^{n}\left(\nabla \cdot\left(\mathbf{u}-\mathbf{u}_{h}\right), w\right)_{\Omega_{i}} & =0,
\end{aligned}
$$

for $\mathbf{v} \in \mathbf{V}_{h, 0}$ and $w \in W_{h}$, where

$$
\sigma(\mathbf{q}, \mathbf{v})=(\mathbf{q}, \mathbf{v})-(\mathbf{q}, \mathbf{v})_{h} .
$$

It was shown in [5] that, $(\mathbf{q}, \mathbf{v})_{h}=0$ for any $\mathbf{v} \in V_{h}$, any constant vector $\mathbf{q}$ and the scalar product (2.4). A similar result has been shown in [6] for the scalar product (2.3). Thus, letting $\mathbf{q}_{0}$ be the mean value of $\mathbf{q}$ on $E$, we get

$$
\left|\sigma(\mathbf{q}, \mathbf{v})_{E}\right|=\left|\sigma\left(\mathbf{q}-\mathbf{q}_{0}, \mathbf{v}\right)_{E}\right| \leq C h|\mathbf{q}|_{1, E}\|\mathbf{v}\|_{0, E}, \quad E \in \mathcal{T}_{h}
$$

Therefore,

$$
\begin{aligned}
\left|\sigma\left(K^{-1} \Pi \mathbf{u}, \mathbf{v}\right)\right| & \leq C \sum_{E \in \mathcal{T}_{h}} h\left\|K^{-1}\right\|_{1, \infty, E}\|\Pi \mathbf{u}\|_{1, E}\|\mathbf{v}\|_{0, E} \\
& \leq C \sum_{i=1}^{n} h\left\|K^{-1}\right\|_{1, \infty, \Omega_{i}}\|\mathbf{u}\|_{1, \Omega_{i}}\|\mathbf{v}\|_{0, \Omega_{i}},
\end{aligned}
$$

using Lemma 5.1 for the last inequality. Clearly (5.17) implies that

$$
\nabla \cdot\left(\Pi_{0} \mathbf{u}-\mathbf{u}_{h}\right)=\nabla \cdot\left(\Pi \mathbf{u}-\mathbf{u}_{h}\right)=0 .
$$

Taking $\mathbf{v}=\Pi_{0} \mathbf{u}-\mathbf{u}_{h}$ in (5.16) we get

$$
\begin{aligned}
\left(K^{-1}\left(\Pi_{0} \mathbf{u}-\mathbf{u}_{h}\right), \Pi_{0} \mathbf{u}-\mathbf{u}_{h}\right)_{h} & \\
= & \sum_{i=1}^{n}\left\langle\mathcal{P}_{h} p-p,\left(\Pi_{0} \mathbf{u}-\mathbf{u}_{h}\right) \cdot \mathbf{n}_{i}\right\rangle_{\Gamma_{i}}+\left(K^{-1}(\Pi \mathbf{u}-\mathbf{u}), \Pi_{0} \mathbf{u}-\mathbf{u}_{h}\right) \\
& +\left(K^{-1}\left(\Pi_{0} \mathbf{u}-\Pi \mathbf{u}\right), \Pi_{0} \mathbf{u}-\mathbf{u}_{h}\right)_{h}-\sigma\left(K^{-1} \Pi \mathbf{u}, \Pi_{0} \mathbf{u}-\mathbf{u}_{h}\right) \\
\leq & \sum_{i=1}^{n}\left\|\mathcal{P}_{h} p-p\right\|_{0, \Gamma_{i}}\left\|\left(\Pi_{0} \mathbf{u}-\mathbf{u}_{h}\right) \cdot \mathbf{n}_{i}\right\|_{0, \Gamma_{i}} \\
& +\left(K^{-1}(\Pi \mathbf{u}-\mathbf{u}), \Pi_{0} \mathbf{u}-\mathbf{u}_{h}\right)+\left(K^{-1}\left(\Pi_{0} \mathbf{u}-\Pi \mathbf{u}\right), \Pi_{0} \mathbf{u}-\mathbf{u}_{h}\right)_{h} \\
& +\left|\sigma\left(K^{-1} \Pi \mathbf{u}, \Pi_{0} \mathbf{u}-\mathbf{u}_{h}\right)\right| \\
\leq & C\left(\sum_{i=1}^{n}\|p\|_{2, \Omega_{i}} h^{3 / 2}\left\|\Pi_{0} \mathbf{u}-\mathbf{u}_{h}\right\|_{0, \Omega_{i}} h^{-1 / 2}\right. \\
& \left.+\sum_{i=1}^{n}\left\|K^{-1}\right\|_{1, \infty, \Omega_{i}}\|\mathbf{u}\|_{1, \Omega_{i}} h\left\|\Pi_{0} \mathbf{u}-\mathbf{u}_{h}\right\|_{0}\right),
\end{aligned}
$$

where we used (5.2), (5.10), (5.4), (5.14), and (5.18) for the last inequality. With (5.19)(5.20), (4.9), (5.5), and (5.15) we have shown the following theorem. 


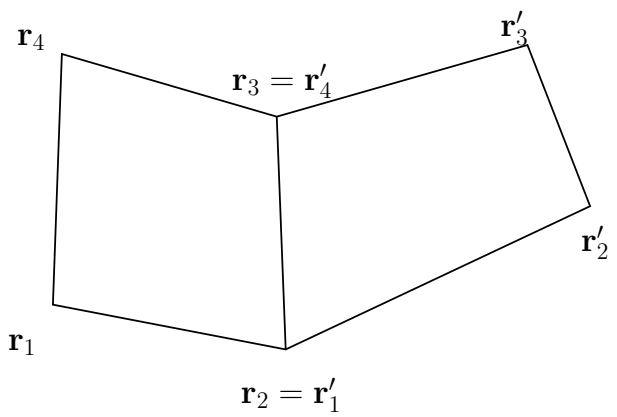

Figure 3: $h^{2}$-uniform quadrilateral grid.

Theorem 5.1 Let $K^{-1} \in W^{1, \infty}\left(\Omega_{i}\right), 1 \leq i \leq n$, and let (4.7) hold. Then, for the velocity $\mathbf{u}_{h}$ of the mortar mimetic finite difference method (4.4)-(4.6), there exists a positive constant $C$ independent of $h$ such that

$$
\left\|\nabla \cdot\left(\mathbf{u}-\mathbf{u}_{h}\right)\right\|_{0} \leq C \sum_{i=1}^{n}\|\mathbf{u}\|_{2, \Omega_{i}} h .
$$

Moreover, if (5.13) holds, then

$$
\left\|\mathbf{u}-\mathbf{u}_{h}\right\|_{0} \leq C \sum_{i=1}^{n}\left(\|p\|_{2, \Omega_{i}}+\|\mathbf{u}\|_{1, \Omega_{i}}\right) h
$$

\subsection{Superconvergence}

In this section, we show that in the case of $h^{2}$-uniform quadrilateral grids, the velocity converges with an order higher than $O(h)$ in a discrete $L^{2}$-norm. It is pertinent to note that the superconvergence result is proved only for the scalar product (2.3).

Referring to Figure 3, a quadrilateral partition is called $h^{2}$-uniform if each element is an $h^{2}$-parallelogram, i.e., $\left\|\left(\mathbf{r}_{2}-\mathbf{r}_{1}\right)-\left(\mathbf{r}_{3}-\mathbf{r}_{4}\right)\right\| \leq C h^{2}$, and any two adjacent quadrilaterals form an $h^{2}$-parallelogram, i.e., $\left\|\left(\mathbf{r}_{2}-\mathbf{r}_{1}\right)-\left(\mathbf{r}_{2}^{\prime}-\mathbf{r}_{1}^{\prime}\right)\right\| \leq C h^{2}$.

To establish the superconvergence, we modify the last inequality in (5.20). In particular, (5.2) gives

$$
\sum_{i=1}^{n}\left\|\mathcal{P}_{h} p-p\right\|_{0, \Gamma_{i}}\left\|\left(\Pi_{0} \mathbf{u}-\mathbf{u}_{h}\right) \cdot \mathbf{n}_{i}\right\|_{0, \Gamma_{i}} \leq C \sum_{i=1}^{n}\|p\|_{5 / 2, \Omega_{i}} h^{2}\left\|\Pi_{0} \mathbf{u}-\mathbf{u}_{h}\right\|_{0, \Omega_{i}} h^{-1 / 2},
$$

and (5.14) gives

$$
\left(K^{-1}\left(\Pi_{0} \mathbf{u}-\Pi \mathbf{u}\right), \Pi_{0} \mathbf{u}-\mathbf{u}_{h}\right)_{h} \leq C \sum_{i=1}^{n}\|\mathbf{u}\|_{3 / 2, \Omega_{i}} h^{3 / 2}\left\|\Pi_{0} \mathbf{u}-\mathbf{u}_{h}\right\|_{0} .
$$

In addition, Theorem 5.1 in [10] implies

$$
\left(K^{-1}(\Pi \mathbf{u}-\mathbf{u}), \Pi_{0} \mathbf{u}-\mathbf{u}_{h}\right) \leq C \sum_{i=1}^{n}\left\|K^{-1}\right\|_{2, \infty, \Omega_{i}}\|\mathbf{u}\|_{2, \Omega_{i}} h^{2}\left\|\Pi_{0} \mathbf{u}-\mathbf{u}_{h}\right\|_{0},
$$


and Lemma 4.3 in [6] gives

$$
\left|\sigma\left(K^{-1} \Pi \mathbf{u}, \Pi_{0} \mathbf{u}-\mathbf{u}_{h}\right)\right| \leq C \sum_{i=1}^{n}\left\|K^{-1}\right\|_{2, \infty, \Omega_{i}}\|\mathbf{u}\|_{2, \Omega_{i}} h^{2}\left\|\Pi_{0} \mathbf{u}-\mathbf{u}_{h}\right\|_{0} .
$$

Combining the above four bounds, we arrive at the following superconvergence result.

Theorem 5.2 Let $K^{-1} \in W^{2, \infty}\left(\Omega_{i}\right), 1 \leq i \leq n$, and let (5.13) hold. Then, for the velocity $\mathbf{u}_{h}$ of the mortar mimetic finite difference method (4.4)-(4.6) with the scalar product (2.3) on $h^{2}$-uniform quadrilateral grids, there exists a positive constant $C$ independent of $h$ such that

$$
\left\|\Pi \mathbf{u}-\mathbf{u}_{h}\right\|_{0} \leq C \sum_{i=1}^{n}\left(\|p\|_{5 / 2, \Omega_{i}}+\|\mathbf{u}\|_{2, \Omega_{i}}\right) h^{3 / 2} .
$$

The above result can be applied to obtain superconvergence for the computed velocity to the average edge fluxes. Define, for any $\mathbf{v} \in\left(H^{1}\left(\Omega_{i}\right)\right)^{2}, i=1, \ldots, n$,

$$
\|\mathbf{v}\|\left\|^{2}=\sum_{E \in \mathcal{T}_{h}}\right\| \mathbf{v}\left\|_{E}^{2}, \quad\right\|\|\mathbf{v}\|_{E}^{2}=\sum_{k=1}^{4}\left(\int_{e_{k}} \mathbf{v} \cdot \mathbf{n}_{k} d s\right)^{2} .
$$

It is easy to see [6] that \|\|$\cdot \|$ is a norm on $\mathbf{V}_{h}$ and there exist constants $c_{1}$ and $c_{2}$ independent of $h$ such that

$$
c_{1}\|\mathbf{v}\|_{0, \Omega} \leq\|\| \mathbf{v}\left\|\leq c_{2}\right\| \mathbf{v} \|_{0, \Omega} \quad \forall \mathbf{v} \in \mathbf{V}_{h} .
$$

Moreover, $\left\||\Pi \mathbf{v}-\mathbf{v} \||=0\right.$ for any $\mathbf{v} \in\left(H^{1}\left(\Omega_{i}\right)\right)^{2}, i=1, \ldots, n$. We have the following superconvergence result.

Theorem 5.3 Under the assumptions of Theorem 5.2, there exists a positive constant $C$ independent of $h$ such that

$$
\left|\left\|\mathbf{u}-\mathbf{u}_{h}\right\|\right| \leq C \sum_{i=1}^{n}\left(\|p\|_{5 / 2, \Omega_{i}}+\|\mathbf{u}\|_{2, \Omega_{i}}\right) h^{3 / 2} .
$$

Proof. By the triangle inequality and (5.22),

$$
\left|\left\|\mathbf{u}-\mathbf{u}_{h}\right\|\right|\left|\leq\left\|\left|\Pi \mathbf{u}-\mathbf{u}_{h}\right|\right\| \leq c_{2}\left\|\Pi \mathbf{u}-\mathbf{u}_{h}\right\|_{0}\right.
$$

and the assertion of the theorem follows from Theorem 5.2.

\section{Pressure error estimates}

In this section we employ a duality argument to obtain a superconvergence estimate for $\mathcal{Q}_{h} p-$ $p_{h}$. The estimate is proved for both scalar products (2.3) and (2.4) on triangular, tetrahedral and $h^{2}$-uniform quadrilateral meshes. The general quadrilateral meshes require the scalar product (2.4). 
Lemma 6.1 Let $K^{-1} \in W^{2, \infty}\left(\Omega_{i}\right), 1 \leq i \leq n$, and the scalar product be given by (2.3). Then, for all $\mathbf{v}, \mathbf{q} \in \mathbf{V}_{h}$, there exists a positive constant $C$ independent of $h$ such that

$$
\left|\sigma\left(K^{-1} \mathbf{v}, \mathbf{q}\right)\right| \leq C \sum_{E \in \mathcal{T}_{h}} h^{r}\|\mathbf{v}\|_{1, E}\|\mathbf{q}\|_{1, E}
$$

where $r=2$ on simplicial elements and $h^{2}$-parallelograms, and $r=1$ on general quadrilateral grids. If the scalar product is given by (2.4), then $r=2$ for both simplicial elements and general quadrilaterals.

Proof. For an element $E \in \mathcal{T}_{h}$, we define the error

$$
\sigma_{E}\left(K^{-1} \mathbf{v}, \mathbf{q}\right)=\int_{E} K^{-1} \mathbf{v} \cdot \mathbf{q} \mathrm{d} \mathbf{x}-\left(K^{-1} \mathbf{v}, \mathbf{q}\right)_{h, E} .
$$

First, we consider the scalar product $\left(K^{-1} \mathbf{v}, \mathbf{q}\right)_{h, E}$ given by (2.3). It was shown in [5] that $\sigma_{E}\left(\mathbf{v}_{0}, \mathbf{q}\right)=0$ for all constant vectors . Using this result and symmetry of (6.1), we get

$$
\begin{aligned}
\sigma_{E}\left(K^{-1} \mathbf{v}, \mathbf{q}\right) & =\sigma_{E}\left(K^{-1} \mathbf{v}, \mathbf{q}-\mathbf{q}_{0}\right)+\sigma_{E}\left(\left(K^{-1}-K_{0}^{-1}\right)\left(\mathbf{v}-\mathbf{v}_{0}\right), \mathbf{q}_{0}\right) \\
& +\sigma_{E}\left(K^{-1} \mathbf{v}_{0}, \mathbf{q}_{0}\right)
\end{aligned}
$$

where $\mathbf{v}_{0}, \mathbf{q}_{0}$ are constant vectors and $K_{0}$ is a constant tensor. By a constant vector (tensor) we mean a vector (tensor) with constant components. Let $\mathbf{v}_{0}$ and $\mathbf{q}_{0}$ be the $L^{2}(E)$ orthogonal projections of $\mathbf{v}$ and $\mathbf{q}$, respectively, onto the space of constant vectors, let $K_{0}^{-1}=K^{-1}\left(m_{E}\right)$, where $m_{E}$ is the center of gravity of $E$, and let $\left(K^{-1} \mathbf{v}\right)_{0}$ be the $L^{2}(E)$ projection of $K^{-1} \mathbf{v}$ into the space of constant vectors. Using the Taylor's theorem, it is easy to verify that

$$
\left\|K^{-1}-K_{0}^{-1}\right\|_{\infty, E} \leq C h\left\|K^{-1}\right\|_{1, \infty, E}
$$

Using (2.5) and (5.3),

$\left|\sigma_{E}\left(K^{-1} \mathbf{v}, \mathbf{q}-\mathbf{q}_{0}\right)\right|=\left|\sigma_{E}\left(K^{-1} \mathbf{v}-\left(K^{-1} \mathbf{v}\right)_{0}, \mathbf{q}-\mathbf{q}_{0}\right)\right| \leq C h^{2}\left\|K^{-1}\right\|_{1, \infty, E}\|\mathbf{v}\|_{1, E}\|\mathbf{q}\|_{1, E}$.

The second term in (6.2) is estimated as follows:

$$
\begin{gathered}
\left|\sigma_{E}\left(\left(K^{-1}-K_{0}^{-1}\right)\left(\mathbf{v}-\mathbf{v}_{0}\right), \mathbf{q}_{0}\right)\right| \leq C\left\|K^{-1}-K_{0}^{-1}\right\|_{\infty, E}\left\|\mathbf{v}-\mathbf{v}_{0}\right\|_{0, E}\left\|\mathbf{q}_{0}\right\|_{0, E} \\
\leq C h^{2}\|K\|_{1, \infty, E}\|\mathbf{v}\|_{1, E}\|\mathbf{q}\|_{0, E} .
\end{gathered}
$$

The remaining term in (6.2) is estimated as follows:

$$
\int_{E} K^{-1} \mathbf{v}_{0} \cdot \mathbf{q}_{0} \mathrm{~d} \mathbf{x}=\overline{K^{-1}} \mathbf{v}_{0} \cdot \mathbf{q}_{0}|E|,
$$

where $\overline{K^{-1}}$ is the mean value of $K^{-1}$ on $E$, and

$$
\left(K^{-1} \mathbf{v}_{0}, \mathbf{q}_{0}\right)_{h, E}=\frac{1}{\alpha_{E}} \sum_{j=1}^{s}\left|T_{j}\right| K^{-1}\left(\mathbf{r}_{j}\right) \mathbf{v}_{0} \cdot \mathbf{q}_{0}
$$

where $\alpha_{E}=2$ for quadrilaterals, $\alpha_{E}=3$ for triangles, $\alpha_{E}=4$ for tetrahedra, and $s$ is the number of vertices of element $E$. For simplicial elements, $\left|T_{j}\right|=|E|$ and it is easy to check 
that the above quadrature is exact for linear tensors. An application of the Bramble-Hilbert lemma gives

$$
\left|\sigma_{E}\left(K^{-1} \mathbf{v}_{0}, \mathbf{q}_{0}\right)\right| \leq C h^{2}\left|K^{-1} \mathbf{v}_{0}\right|_{2, E}\left\|\mathbf{q}_{0}\right\|_{0, E} \leq C h^{2}\left|K^{-1}\right|_{2, \infty, E}\left\|\mathbf{v}_{0}\right\|_{0, E}\left\|\mathbf{q}_{0}\right\|_{0, E} .
$$

For general quadrilaterals, the quadrature is exact for constant tensors and we have

$$
\left|\sigma_{E}\left(K^{-1} \mathbf{v}_{0}, \mathbf{q}_{0}\right)\right|=\left|\sigma_{E}\left(\left(K^{-1}-K_{0}^{-1}\right) \mathbf{v}_{0}, \mathbf{q}_{0}\right)\right| \leq C h\left\|K^{-1}\right\|_{1, \infty, E}\left\|\mathbf{v}_{0}\right\|_{0, E}\left\|\mathbf{q}_{0}\right\|_{0, E}
$$

Let us show that this term is $O\left(h^{2}\right)$ in the case of $h^{2}$-parallelograms. To do this we map it to the reference element. It follows from (3.5) that $J_{E}\left(\hat{\mathbf{r}}_{j}\right)=2\left|T_{j}\right|$. Thus,

$$
\begin{aligned}
\left(K^{-1} \mathbf{v}_{0}, \mathbf{q}_{0}\right)_{h, E} & =\frac{1}{2} \sum_{j=1}^{4}\left|T_{j}\right| K^{-1}\left(\mathbf{r}_{j}\right) \mathbf{v}_{0} \cdot \mathbf{q}_{0}=\frac{1}{2} \sum_{j=1}^{4}\left|T_{j}\right| \hat{K}^{-1}\left(\hat{\mathbf{r}}_{j}\right) \mathbf{v}_{0} \cdot \mathbf{q}_{0} \\
& =\frac{1}{4} \sum_{j=1}^{4} B_{E}\left(\hat{\mathbf{r}}_{j}\right) \mathbf{v}_{0} \cdot \mathbf{q}_{0} \equiv\left(B_{E} \mathbf{v}_{0}, \mathbf{q}_{0}\right)_{T},
\end{aligned}
$$

where $B_{E}=J_{E} \hat{K}^{-1}$. Note that the quadrature rule $(\cdot, \cdot)_{T}$ is the trapezoidal rule on the reference square $\hat{E}$.

For the integral term in the quadrature error we write

$$
\int_{E} K^{-1} \mathbf{v}_{0} \cdot \mathbf{q}_{0} \mathrm{~d} \mathbf{x}=\int_{\hat{E}} \hat{K}^{-1} \mathbf{v}_{0} \cdot \mathbf{q}_{0} J_{E} \mathrm{~d} \hat{\mathbf{x}}=\int_{\hat{E}} B_{E} \mathbf{v}_{0} \cdot \mathbf{q}_{0} \mathrm{~d} \hat{\mathbf{x}} .
$$

Using (6.5) and (6.6) we obtain

$$
\sigma_{E}\left(K^{-1} \mathbf{v}_{0}, \mathbf{q}_{0}\right)=\int_{\hat{E}} B_{E} \mathbf{v}_{0} \cdot \mathbf{q}_{0} \mathrm{~d} \hat{\mathbf{x}}-\left(B_{E} \mathbf{v}_{0}, \mathbf{q}_{0}\right)_{T} \equiv \sigma_{\hat{E}}\left(B_{E} \mathbf{v}_{0}, \mathbf{q}_{0}\right) .
$$

Since the trapezoidal quadrature rule on $\hat{E}$ is exact for linear polynomials, the Bramble-Hilbert lemma implies that

$$
\left|\sigma_{\hat{E}}\left(B \mathbf{v}_{0}, \mathbf{q}_{0}\right)\right| \leq C|B|_{2, \infty, \hat{E}}\left\|\mathbf{v}_{0}\right\|_{0, \hat{E}}\left\|\mathbf{q}_{0}\right\|_{0, \hat{E}}
$$

To bound on $|B|_{2, \infty, \hat{E}}$, we note that for an $h^{2}$-parallelogram

$$
\left|J_{E}\right|_{1, \infty, \hat{E}} \leq C h^{3}, \quad\left|J_{E}\right|_{2, \infty, \hat{E}}=0, \quad\left|F_{E}\right|_{s, \infty, \hat{E}} \leq C h^{s}, s=1,2 .
$$

Therefore,

$$
|B|_{2, \infty, \hat{E}} \leq C\left(h^{3}\left|\hat{K}^{-1}\right|_{1, \infty, \hat{E}}+h^{2}\left|\hat{K}^{-1}\right|_{2, \infty, \hat{E}}\right) \leq C h^{4}\left\|K^{-1}\right\|_{2, \infty, E},
$$

using the chain rule for the last inequality. The above bound, combined with (6.7) and (6.8), implies

$$
\begin{aligned}
\left|\sigma_{E}\left(K^{-1} \mathbf{v}_{0}, \mathbf{q}_{0}\right)\right| & \leq C h^{4}\left\|K^{-1}\right\|_{2, \infty, E}\left\|\mathbf{v}_{0}\right\|_{0, \hat{E}}\left\|\mathbf{q}_{0}\right\|_{0, \hat{E}} \\
& \leq C h^{2}\left\|K^{-1}\right\|_{2, \infty, E}\|\mathbf{v}\|_{0, E}\|\mathbf{q}\|_{0, E},
\end{aligned}
$$

Let the scalar product $\left(K^{-1} \mathbf{v}, \mathbf{q}\right)_{h, E}$ be given by (2.4). The only thing we have to do is to derive an estimate for the third term in (6.2). Note that the scalar product

$$
\left(K^{-1} \mathbf{v}_{0}, \mathbf{q}_{0}\right)_{h, E}=\frac{1}{\alpha_{E}} \sum_{j=1}^{s}\left|T_{j}\right| K_{E}^{-1} \mathbf{v}_{0} \cdot \mathbf{q}_{0}=|E| K_{E}^{-1} \mathbf{v}_{0} \cdot \mathbf{q}_{0}
$$


is exact for linear tensors for both simplicial and quadrilateral elements. The application of the Bramble-Hilbert lemma gives estimate (6.4).

The assertion of the lemma follows from the Hölder inequality.

We continue with the duality argument for bounding $\left\|\mathcal{Q}_{h} p-p_{h}\right\|_{0}$. We first rewrite the error equation (5.16) as follows:

$$
\left(K^{-1}\left(\mathbf{u}-\mathbf{u}_{h}\right), \mathbf{v}\right)=\sum_{i=1}^{n}\left(\left(p-p_{h}, \nabla \cdot \mathbf{v}\right)_{\Omega_{i}}-\left\langle p, \mathbf{v} \cdot \mathbf{n}_{i}\right\rangle_{\Gamma_{i}}\right)-\sigma\left(K^{-1} \mathbf{u}_{h}, \mathbf{v}\right)
$$

Let $\varphi$ be the solution of

$$
\begin{aligned}
-\nabla \cdot K \nabla \varphi & =-\left(\mathcal{Q}_{h} p-p_{h}\right) & & \text { in } \Omega, \\
\varphi & =0 & & \text { on } \partial \Omega .
\end{aligned}
$$

By elliptic regularity,

$$
\|\varphi\|_{2} \leq C\left\|\mathcal{Q}_{h} p-p_{h}\right\|_{0}
$$

Take $\mathbf{v}=\Pi_{0} K \nabla \varphi$ in (6.9) to get

$$
\begin{aligned}
\left\|\mathcal{Q}_{h} p-p_{h}\right\|_{0}^{2}= & \sum_{i=1}^{n}\left(\mathcal{Q}_{h} p-p_{h}, \nabla \cdot \Pi_{0} K \nabla \varphi\right)_{\Omega_{i}} \\
= & \sum_{i=1}^{n}\left(\left(K^{-1}\left(\mathbf{u}-\mathbf{u}_{h}\right), \Pi_{0} K \nabla \varphi\right)_{\Omega_{i}}+\left\langle p-\mathcal{P}_{h} p, \Pi_{0} K \nabla \varphi \cdot \mathbf{n}_{i}\right\rangle_{\Gamma_{i}}\right) \\
& +\sigma\left(K^{-1} \mathbf{u}_{h}, \Pi_{0} K \nabla \varphi\right) .
\end{aligned}
$$

The first two terms on the right in (6.11) appear also in the proof of Theorem 5.1 in [1], where it was shown that

$$
\begin{aligned}
& \sum_{i=1}^{n}\left(\left(K^{-1}\left(\mathbf{u}-\mathbf{u}_{h}\right), \Pi_{0} K \nabla \varphi\right)_{\Omega_{i}}+\left\langle p-\mathcal{P}_{h} p, \Pi_{0} K \nabla \varphi \cdot \mathbf{n}_{i}\right\rangle_{\Gamma_{i}}\right) \\
\leq & C \sum_{i=1}^{n} h^{2}\|K\|_{1, \infty, \Omega_{i}}\left(\|p\|_{2, \Omega_{i}}+\|\mathbf{u}\|_{2, \Omega_{i}}\right)\|\varphi\|_{2, \Omega_{i}} .
\end{aligned}
$$

Using Lemma 6.1, the last term in (6.11) can be bounded as

$$
\begin{aligned}
& \left|\sigma\left(K^{-1} \mathbf{u}_{h}, \Pi_{0} K \nabla \varphi\right)\right| \\
& \leq C \sum_{E \in \mathcal{T}_{h}} h^{r}\left\|\mathbf{u}_{h}\right\|_{1, E}\left\|\Pi_{0} K \nabla \varphi\right\|_{1, E} \\
& \leq C \sum_{E \in \mathcal{T}_{h}} h^{r}\left(\left\|\mathbf{u}_{h}-\Pi \mathbf{u}\right\|_{1, E}+\|\Pi \mathbf{u}\|_{1, E}\right) \\
& \quad \times\left(\left\|\Pi_{0} K \nabla \varphi-\Pi K \nabla \varphi\right\|_{1, E}+\|\Pi K \nabla \varphi\|_{1, E}\right) \\
& \leq C \sum_{E \in \mathcal{T}_{h}} h^{r}\left(h^{-1}\left\|\mathbf{u}_{h}-\Pi \mathbf{u}\right\|_{0, E}+\|\mathbf{u}\|_{1, E}\right) \\
& \quad \times\left(h^{-1}\left\|\Pi_{0} K \nabla \varphi-\Pi K \nabla \varphi\right\|_{0, E}+\|K \nabla \varphi\|_{1, E}\right) \\
& \leq C \sum_{i=1}^{n} h^{r}\|K\|_{1, \infty, \Omega_{i}}\left(\|p\|_{2, \Omega_{i}}+\|\mathbf{u}\|_{1, \Omega_{i}}\right)\|\varphi\|_{2, \Omega_{i}},
\end{aligned}
$$

where we used the inverse inequality and Lemma 5.1 in the third inequality, and Theorem 5.1 and (5.14) in the last inequality. A combination of (6.10)-(6.13) gives the following result. 
Theorem 6.1 Let $K \in W^{1, \infty}\left(\Omega_{i}\right)$ and $K^{-1} \in W^{2, \infty}\left(\Omega_{i}\right), 1 \leq i \leq n$, and the scalar product be given by (2.3). Then, for the pressure $p_{h}$ of the mortar mimetic finite difference method (4.4)-(4.6), if (5.13) holds, then

$$
\begin{aligned}
\left\|\mathcal{Q}_{h} p-p_{h}\right\|_{0} & \leq C \sum_{i=1}^{n}\left(\|p\|_{2, \Omega_{i}}+\|\mathbf{u}\|_{2, \Omega_{i}}\right) h^{r}, \\
\left\|p-p_{h}\right\|_{0} & \leq C \sum_{i=1}^{n}\left(\|p\|_{2, \Omega_{i}}+\|\mathbf{u}\|_{2, \Omega_{i}}\right) h
\end{aligned}
$$

where $r=2$ on meshes with both simplicial elements and $h^{2}$ parallelograms, and $r=1$ on general quadrilateral grids. If the scalar product is given by (2.4), then $r=2$ on meshes with both simplicial elements and general quadrilaterals.

\section{Numerical experiments}

In this section we confirm the theoretical estimates for a special case of non-matching meshes, the locally refined meshes. An example of a computational mesh is shown in Fig. 4. The mesh consists of 13 quadrilateral subdomains with different levels of uniform refinement. The convergence of the mortar MFD method has been studied on the sequence of meshes generated by uniform refinement (and coarsening) of the shown mesh.

Another sequence of meshes is generated from the above sequence by perturbing the positions of mesh nodes. The mesh node is moved to a random position inside a square centered at the node. The side of the square are aligned with the coordinate axes and equal to $40 \%$ of the size of the smallest edge sharing the node. The mesh nodes on the domain boundary and subdomain interfaces are not perturbed. An example of a random mesh is shown in Fig. 5.
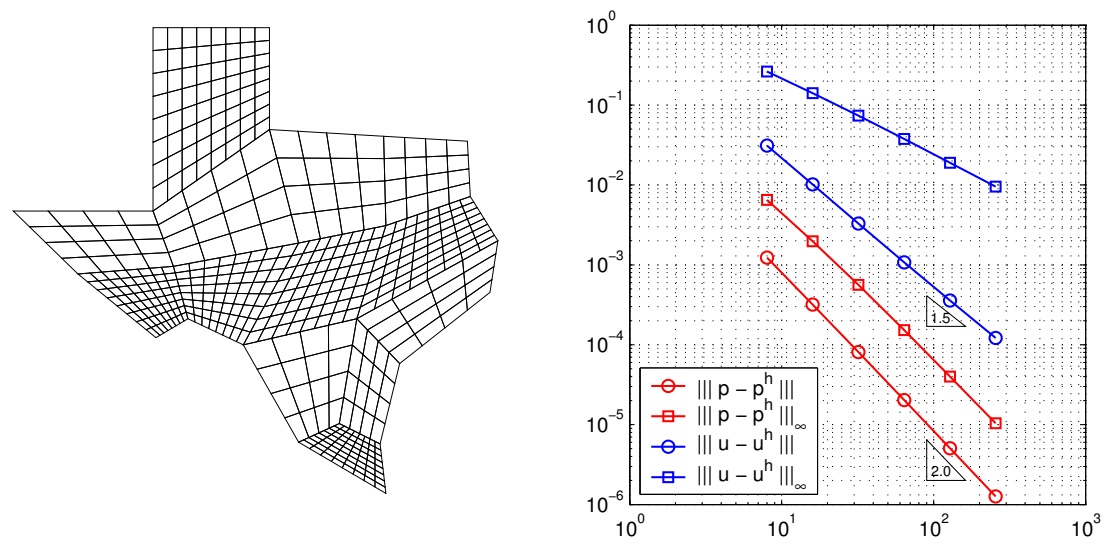

Figure 4: Convergence rates on a smooth mesh

The interface conditions for the mortar MFD method are drastically simplified in the case of locally refined meshes. Let us consider the interface $\Gamma_{i, j}$. Let $\mathcal{E}_{h, i, j}$ be the finer partition, $\tilde{\mathcal{E}}_{h, i, j}=\mathcal{E}_{h, j, i}$ and the mortar space $\Lambda_{h, i, j}$ be discontinuous.

In order to describe projectors $R_{i, j}$ and $R_{j, i}$, it is sufficient to consider a three-cell interface. Let cells $E_{1}$ and $E_{2}$ from $\mathcal{T}_{h, i}$ be adjacent to a cell $E_{3}$ from $\mathcal{T}_{h, j}$. Without loss of generality, we assume that $\mathcal{E}_{h, i, j}=\left\{f_{1}, f_{2}\right\}$ and $\mathcal{E}_{h, j, i}=\left\{f_{3}\right\}$. Then, the dimension of the 

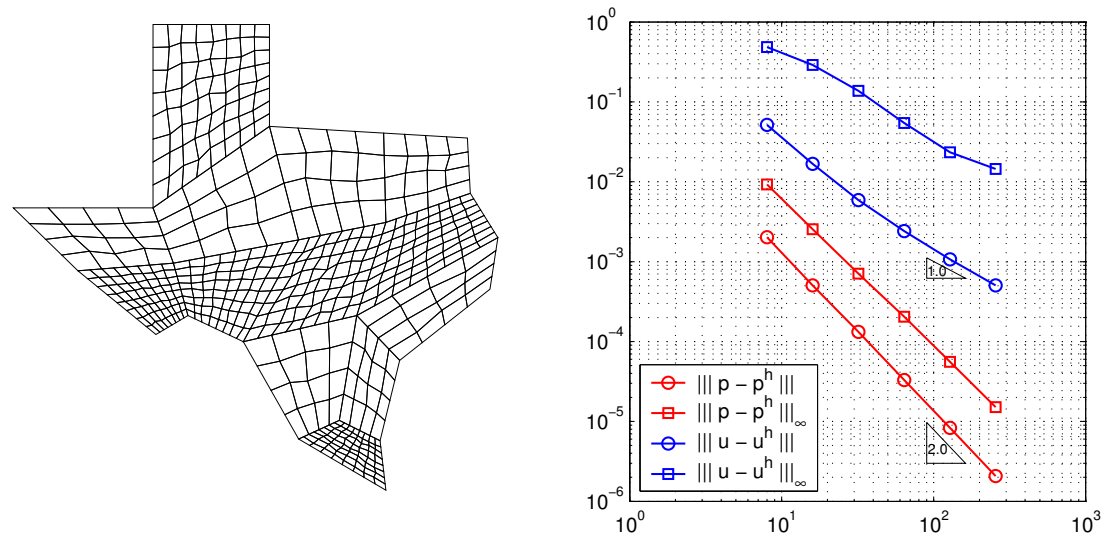

Figure 5: Convergence rates on a random mesh

mortar space is $2, R_{i, j}$ is a $2 \times 2$ matrix, and $R_{j, i}$ is a $1 \times 2$ matrix. It is easy to check that definition (4.1) results in

$$
R_{i, i}=\frac{1}{2\left|f_{3}\right|}\left[\begin{array}{cc}
\left|f_{1}\right| & \left|f_{2}\right|+\left|f_{3}\right| \\
\left|f_{1}\right|+\left|f_{3}\right| & \left|f_{2}\right|
\end{array}\right] \quad \text { and } \quad R_{j, i}=\frac{1}{2}\left[\begin{array}{ll}
1 & 1
\end{array}\right] .
$$

Eliminating vector $\vec{\lambda}$ from (2.13), we get the following continuity conditions:

$$
\left|f_{1}\right| p_{f_{1}}+\left|f_{2}\right| p_{f_{2}}=\left|f_{3}\right| p_{f_{3}} \quad \text { and } \quad u_{f_{1}}=u_{f_{2}}=-u_{f_{3}} .
$$

Similar condition has been proposed and analyzed numerically in [13]. It has been shown that the resulting MFD method is exact for linear solutions.

Let $p(x, y)=x^{3} y^{2}+x \cos (x y) \sin (x)$ be the exact solution and $K$ be the full tensor,

$$
K(x, y)=\left(\begin{array}{cc}
(x+1)^{2}+y^{2} & -x y \\
-x y & (x+1)^{2}
\end{array}\right) .
$$

The right pictures in Fig. 4 and 5 show the convergence rates for the pressure and velocity. In addition to norm (5.21), we show the convergence rate in the discrete $L_{\infty}$-norm:

$$
\left\|\left|\mathbf{u}-\mathbf{u}_{h}\right|\right\|_{\infty}=\max _{f}\left|\frac{1}{|f|} \int_{f} \mathbf{u} \cdot \mathbf{n}_{f} d s-\mathbf{u}_{h} \cdot \mathbf{n}_{f}\right|,
$$

where maximum is taken over all mesh edges. The convergence rates for the pressure variable are shown in the following discrete norms:

$$
\|\left.\left|p-p^{h}\right|\right|^{2}=\sum_{e_{i} \in \mathcal{T}_{h}}\left|p\left(c_{i}\right)-p_{h}\left(c_{i}\right)\right|^{2}\left|e_{i}\right|
$$

and

$$
||\left|p-p^{h}\right|||_{\infty}=\max _{e_{i} \in \mathcal{T}_{h}}\left|p\left(c_{i}\right)-p_{h}\left(c_{i}\right)\right|,
$$

where $c_{i}$ is the geometric center of element $e_{i}$. The use of the geometric center instead of the mass center is due to the following property of the mortar MFD method. The method is exact 
for linear solutions when the pressure variable, $p\left(c_{i}\right)$, is evaluated at the geometric center $c_{i}$ [5].

The mortar MFD method with the scalar product (2.3) has been used on the smooth meshes. The convergence rate for the velocity variable is 1.6. This is slightly higher than the theoretically predicted rate of 1.5 (see Theorem 5.2). The second order convergence rate for the pressure variable confirms the assertion of Theorem 6.1.

The mortar MFD method with the scalar product (2.4) has been used on the random meshes. The asymptotically optimal convergence rate for the velocity is in agreement with the assertion of Theorem 5.1. The second order convergence rate for the pressure variable was theoretically predicted in Theorem 6.1.

\section{References}

[1] T. Arbogast, L. C. Cowsar, M. F. Wheeler, and I. Yotov. Mixed finite element methods on nonmatching multiblock grids. SIAM J. Numer. Anal., 37(4):1295-1315, 2000.

[2] T. Arbogast, C. N. Dawson, P. T. Keenan, M. F. Wheeler, and I. Yotov. Enhanced cell-centered finite differences for elliptic equations on general geometry. SIAM J. Sci. Comp., 19(2):404-425, 1998.

[3] F. Ben Belgacem. The mortar finite element method with Lagrange multipliers. Numer. Math., 84(2):173-197, 1999.

[4] C. Bernardi, Y. Maday, and A. T. Patera. A new nonconforming approach to domain decomposition: the mortar element method. In H. Brezis and J. L. Lions, editors, Nonlinear partial differential equations and their applications. Longman Scientific \& Technical, UK, 1994.

[5] M. Berndt, K. Lipnikov, J. D. Moulton, and M. Shashkov. Convergence of mimetic finite difference discretizations of the diffusion equation. East-West J. Numer. Math., 9:253-284, 2001.

[6] M. Berndt, K. Lipnikov, M. Shashkov, M. F. Wheeler, and I. Yotov. Superconvergence of the velocity in mimetic finite difference methods on quadrilaterals. submitted, 2004.

[7] F. Brezzi and M. Fortin. Mixed and hybrid finite element methods. Springer-Verlag, New York, 1991.

[8] J. Campbell and M. Shashkov. A tensor artificial viscosity using a mimetic finite difference algorithm. J. Comput. Phys., 172:739-765, 2001.

[9] P. G. Ciarlet. The finite element method for elliptic problems. North-Holland, New York, 1978.

[10] R. E. Ewing, M. Liu, and J. Wang. Superconvergence of mixed finite element approximations over quadrilaterals. SIAM J. Numer. Anal., 36(3):772-787, 1999.

[11] P. Grisvard. Elliptic problems in nonsmooth domains. Pitman, Boston, 1985.

[12] J. M. Hyman, M. Shashkov, and S. Steinberg. The numerical solution of diffusion problems in strongly heterogeneous non-isotropic materials. J. Comput. Phys., 132:130-148, 1997.

[13] K. Lipnikov, J. Morel, and M. Shashkov. Mimetic finite difference methods for diffusion equations on non-orthogonal non-conformal meshes. J. Comput. Phys., (in press), 2004. 
[14] L. Margolin, M. Shashkov, and P. Smolarkiewicz. A discrete operator calculus for finite difference approximations. Comput. Meth. Appl. Mech. Engrg., 187:365-383, 2000.

[15] J. E. Morel, R. M. Roberts, and M. Shashkov. A local support-operators diffusion disretization scheme for quadrilateral $r-z$ meshes. J. Comput. Phys., 144:17-51, 1998.

[16] J. C. Nedelec. Mixed finite elements in $\mathbf{R}^{3}$. Numer. Math., 35:315-341, 1980.

[17] R. A. Raviart and J. M. Thomas. A mixed finite element method for 2nd order elliptic problems. In Mathematical Aspects of the Finite Element Method, Lecture Notes in Mathematics, volume 606, pages 292-315. Springer-Verlag, New York, 1977.

[18] J. E. Roberts and J.-M. Thomas. Mixed and hybrid methods. In P. G. Ciarlet and J.L. Lions, editors, Handbook of Numerical Analysis, Vol. II, pages 523-639. Elsevier Science Publishers B.V., 1991.

[19] M. Shashkov and S. Steinberg. Solving diffusion equations with rough coefficients in rough grids. J. Comput. Phys., 129:383-405, 1996.

[20] J. M. Thomas. Sur l'analyse numérique des méthods d'éléments finis hybrides et mixtes. $\mathrm{PhD}$ thesis, Université Pierre et Marie Curie, Paris, 1977.

[21] J. Wang and T. P. Mathew. Mixed finite element method over quadrilaterals. In I. T. Dimov, B. Sendov, and P. Vassilevski, editors, Conference on Advances in Numerical Methods and Applications, pages 203-214. World Scientific, River Edge, NJ, 1994.

[22] Barbara I. Wohlmuth. A mortar finite element method using dual spaces for the Lagrange multiplier. SIAM Journal on Numerical Analysis, 38(3):989-1012, 2000.

[23] I. Yotov. Mixed finite element methods for flow in porous media. PhD thesis, Rice University, Houston, Texas, 1996. TR96-09, Dept. Comp. Appl. Math., Rice University and TICAM report 96-23, University of Texas at Austin. 